\title{
Intersection cuts from multiple rows: a disjunctive programming approach
}

\author{
Egon Balas • Andrea Qualizza
}

Received: 1 May 2012 / Accepted: 8 January 2013 / Published online: 4 April 2013

(C) Springer-Verlag Berlin Heidelberg and EURO - The Association of European Operational Research Societies 2013

\begin{abstract}
We address the issue of generating cutting planes for mixed integer programs from multiple rows of the simplex tableau with the tools of disjunctive programming. A cut from $q$ rows of the simplex tableau is an intersection cut from a $q$-dimensional parametric cross-polytope, which can also be viewed as a disjunctive cut from a $2^{q}$-term disjunction. We define the disjunctive hull of the $q$-row problem, describe its relation to the integer hull, and show how to generate its facets. For the case of binary basic variables, we derive cuts from the stronger disjunctions whose terms are equations. We give cut strengthening procedures using the integrality of the nonbasic variables for both the integer and the binary case. Finally, we discuss some computational experiments.
\end{abstract}

Keywords Mixed integer programming - Disjunctive programming - Intersection cuts $\cdot 0-1$ programming

Mathematics Subject Classification $90 \mathrm{C} 10 \cdot 90 \mathrm{C} 11 \cdot 90 \mathrm{C} 27$

\section{Introduction: intersection cuts and disjunctive programming}

In the last few years considerable effort has been devoted to generating valid cuts for mixed integer programs from multiple rows of the simplex tableau, with a focus on

Research supported by NSF Grant \#DMI-352885 and ONR contract \#N00014-03-1-0133.

\section{E. Balas $(\varangle)$}

Tepper School of Business, Carnegie Mellon University, Pittsburgh, PA 15213, USA

e-mail: eb17@andrew.cmu.edu

Present Address:

A. Qualizza

Amazon Research, Seattle, WA 98109, USA 
cuts from two rows. This research was pioneered by the 2007 paper of Andersen et al. (2007), followed by Borozan and Cornuéjols (2009), Cornuéjols and Margot (2009), Dey and Wolsey (2010) and many others [Basu et al. 2009, 2010; Dash et al. 2010; Dey et al. 2010; for a recent survey see Conforti et al. (2011)].

All of these papers view and derive the multiple-row cuts as intersection cuts, a concept introduced in Balas (1971), i.e. cuts obtained by intersecting the extreme rays of the cone defined by a basic linear programming solution with the boundary of a convex set whose interior contains no feasible integer point. Intersection cuts can be viewed as disjunctive cuts, and in this paper we apply the tools of disjunctive programming to the study of cuts from multiple rows of the simplex tableau. Two early versions of this paper were presented at the 2009 Spring Meeting of the AMS in San Francisco (Balas 2009a) and at the 20th ISMP in Chicago (Balas 2009b). Among the papers cited above, Dash et al. (2010) also addresses two-row cuts from a disjunctive point of view.

The structure of our paper is as follows. In the remainder of this section we outline the connection of intersection cuts with disjunctive programming. In Sec. 2 we introduce the concept of disjunctive hull associated with $q$ rows of the simplex tableau and examine the relation between the disjunctive hull and the integer hull. We then give a geometric interpretation of cuts from $q$ rows of the simplex tableau as cuts from a $q$-dimensional parametric cross-polytope (Sec. 3), followed by a theorem relating the facets of the disjunctive hull to those of the integer hull (Sec. 4). In Sec. 5 we specialize these results to the case of $q=2$. The next section (6) discusses the strengthening of our cuts when some of the nonbasic variables are integer-constrained. Section 7 deals with the 0-1 case, when the stronger disjunction whose terms are equations can be used to derive stronger cuts. Finally, Sec. 8 describes some computational experiments.

Consider a Mixed Integer Program with linear constraint set $A x \geq b, x \in \mathbb{R}^{n}$, and an associated simplex tableau

$$
x=\bar{x}+\sum_{j \in J} r^{j} s_{j}, \quad x \in \mathbb{Z}_{+}^{q}, s \in \mathbb{R}_{+}^{n} .
$$

Here $\bar{x}$ is a basic feasible solution to LP, the linear programming relaxation of a MIP, and we are interested in generating an inequality that cuts off $\bar{x}$ but no feasible integer point.

Theorem 1.1 (Balas 1971) Let $T \subseteq \mathbb{R}^{q}$ be a closed convex set whose interior contains $\bar{x}$ but no feasible integer point. For $j \in J$, let $s_{j}^{*}:=\max \left\{s_{j}: \bar{x}+r^{j} s_{j} \in T\right\}$. Then the inequality $\alpha s \geq 1$, where $\alpha_{j}=\frac{1}{s_{j}^{*}}, j \in J$, cuts off $\bar{x}$ but no feasible integer point.

The inequality $\alpha s \geq 1$ is known as an intersection cut.

Theorem 1.1 is illustrated by Fig. 1. In both cases (a) and (b) the convex set $T$ consists of the intersection of two halfspaces, but in (b) the two halfspaces are defined by hyperplanes parallel to one of the coordinate axes, and so their intersection defines an infinite strip. The intersection cut from this latter set $T$ is the Gomory mixed integer cut (GMI) (Gomory 1960). 


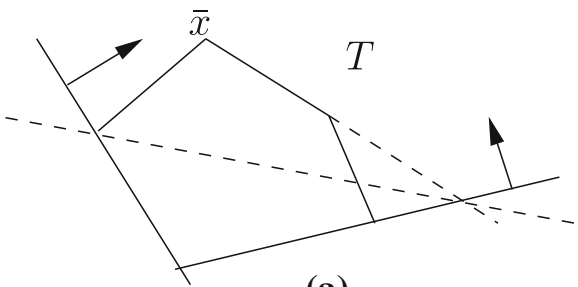

(a)

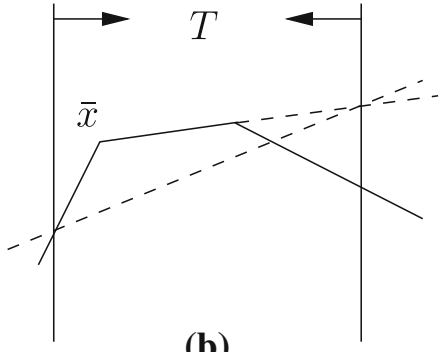

(b)

Fig. 1 Two intersection cuts

This particular class of intersection cuts, the GMI cuts, has played a crucial role in making mixed integer programs practically solvable. These cuts are derived from a convex set of the form $\left\lfloor\bar{x}_{i}\right\rfloor \leq x_{i} \leq\left\lceil\bar{x}_{i}\right\rceil$, where $x_{i}=\bar{x}_{i}+\sum_{j \in J} r_{j}^{i} s_{j}$ is one of the rows of an optimal simplex tableau and $\left\lfloor\bar{x}_{i}\right\rfloor<\bar{x}_{i}<\left\lceil\bar{x}_{i}\right\rceil$. More generally, cuts obtained from a convex set of the form $\pi_{0} \leq \pi x \leq \pi_{0}+1$, where $\left(\pi, \pi_{0}\right)$ is an integer vector, are known in the literature as split cuts (Cook et al. 1990). It is then natural to ask the question whether intersection cuts derived simultaneously from several rows of a simplex tableau have some properties that distinguish them from split cuts. It was this question that has led to the investigation of intersection cuts from maximal lattice-free convex sets by Andersen et al. (2007); Borozan and Cornuéjols (2009) and others.

We propose a different approach to the same problem, which promises some computational advantages. The approach is that of Disjunctive Programming, a natural outgrowth of the study of intersection cuts. To see the connection, consider an intersection cut from a cone with apex at $\bar{x}$ of (1.1) and a polyhedral set $T:=\left\{x: d^{i} x \leq d_{0}^{i}, i=1, \ldots, m\right\}$ such that $\bar{x} \in \operatorname{int} T$ and int $T$ contains no feasible integer point. Clearly, this latter condition can be rephrased as the requirement that every integer point satisfying (1.1) should satisfy at least one of the weak complements of the inequalities defining $T$, i.e. should satisfy the disjunction

$$
\bigvee_{i=1}^{m}\left(d^{i} x \geq d_{0}^{i}\right)
$$

Therefore an intersection cut from (1.1) and $T$ can be viewed as a disjunctive cut from (1.1) and (1.2). In fact, the cut $\delta x \geq 1$ from (1.1) and the disjunction (1.2) has coefficients

$$
\delta_{j}=\max _{i \in\{1, \ldots, m\}}\left\{\frac{d_{j}^{i}}{d_{0}^{i}}\right\}, \quad j=1, \ldots, n,
$$

and it is easy to see that $\delta_{j}$ is the same as $\alpha_{j}$ of Theorem 1.1 for the case when $T$ is as above.

On the other hand, the disjunctive point of view opens up new perspectives. Thus it is easy to see that the disjunction (1.2) remains valid if each of its terms gets augmented with any inequalities valid for all of them. Thus, if we introduce into each term of (1.2) all the valid inequalities for (1.1), namely $A x \geq b$, we get the valid disjunction 


$$
\bigvee_{i=1}^{m}\left(\begin{array}{c}
A x \geq b \\
d^{i} x \geq d_{0}^{i}
\end{array}\right)
$$

Now (1.4) cuts off more points than (1.1) and (1.2), since (1.1) is just a particular basic solution of $A x \geq b$. In fact, an easy way to derive a valid cut from (1.4) is to amalgamate the constraints of each term into a single inequality, i.e. replace (1.4) with

$$
\bigvee_{i=1}^{m}\left(\left(u^{i} A+u_{0}^{i} d^{i}\right) x \geq u^{i} b+u_{0}^{i} d_{0}^{i}\right)
$$

for some vectors $\left(u^{i}, u_{0}^{i}\right) \geq 0, i=1, \ldots, m$, and apply the above formula to the resulting disjunction (1.5), to obtain the valid inequality $\beta(u) x \geq 1$, where

$$
\beta(u)_{j}=\max _{i \in\{1, \ldots, m\}}\left\{\frac{u^{i} a_{j}^{i}+u_{0}^{i} d_{j}^{i}}{u^{i} b+u_{0}^{i} d_{0}^{i}}:\left(u^{i}, u_{0}^{i}\right) \geq 0\right\}, \quad j=1, \ldots, n .
$$

Thus (1.4) yields not a single inequality, but a family of inequalities, each value assignment to the vectors $\left(u^{i}, u_{0}^{i}\right)$ yielding a particular one. Clearly, (1.6) defines a stronger inequality than (1.3), the latter corresponding to the special case of the former when $\left(u^{i}, u_{0}^{i}\right)=(0,1)$ for all $i \in\{1, \ldots, m\}$.

If we now are interested in a polyhedron which can yield an intersection cut equivalent to a disjunctive cut from (1.4), we find that there is no single polyhedron, but a family of polyhedra parameterized by the vectors $\left(u^{i}, u_{0}^{i}\right)$, obtained by reversing the inequalities (1.5):

$$
T(u):=\left\{x:\left(u^{i} A+u_{0}^{i} d^{i}\right) x \leq u^{i} b+u_{0}^{i} d_{0}^{i}, \quad i=1, \ldots, m\right\} .
$$

In this way every disjunctive cut from (1.4) can be viewed as an intersection cut obtained from a parameterized polyhedron $T(u)$.

We illustrate this discussion on a two-term disjunction. Given an arbitrary mixed integer set of the form (1.1), let $\left(\pi, \pi_{0}\right)$ be an integer vector with a component $\pi_{j}$ for every integer-constrained variable. Then the disjunctive cut derived from

$$
\pi x \leq \pi_{0} \vee \pi x \geq \pi_{0}+1
$$

is equivalent to the intersection cut derived from the convex set

$$
\pi_{0} \leq \pi x \leq \pi_{0}+1
$$

illustrated in Fig. 1. On the other hand, the disjunction

$$
\left(\begin{array}{l}
A x \geq b \\
\pi x \leq \pi_{0}
\end{array}\right) \vee\left(\begin{array}{l}
A x \geq b \\
\pi x \geq \pi_{0}+1
\end{array}\right)
$$


gives rise to an entire family of cuts, whose members are determined by the multipliers $\left(u, u_{0}\right),\left(v, v_{0}\right) \geq 0$ associated with the constraints of this more general disjunction, namely

$$
\left(u_{0} \pi-u A\right) x \leq u_{0} \pi_{0}-u b \vee\left(v_{0} \pi+v A\right) x \geq v_{0} \pi_{0}+v b+1 .
$$

To see that (1.9) is stronger than (1.7) it is sufficient to remark that any $x$ that satisfies (1.7) but violates the $i$ th constraint of $A x \geq b$ also violates those members of the family (1.9) having a sufficiently large $u_{i}$ or $v_{i}$ component. Cuts derived from a disjunction of the form (1.7) are called split cuts, a term that reflects the fact that (1.7) splits the space into two disjoint half-spaces. Cook et al. (1990) who coined this term also extended it to the larger family of cuts derived from disjunctions of the form (1.9).

Disjunctive sets of the form (1.8) represent unions of polyhedra, and the study of optimization over unions of polyhedra is known as Disjunctive Programming. Its two basic results are a compact representation of the convex hull of a union of polyhedra in a higher dimensional space, and the sequential convexifiability of facial disjunctive sets (Balas 1979, 1998). The application of disjunctive programming to mixed 0-1 programs has become known as the lift-and-project method (Balas et al. 1993). Here we apply this approach to the study of intersection cuts from multiple rows of the simplex tableau.

\section{Integer and disjunctive hulls}

Consider again a system defined by $q$ rows of the simplex tableau, this time without the integrality constraints:

$$
x=f+\sum_{j \in J} r^{j} s_{j}, s_{j} \geq 0, j \in J,
$$

where $f, r^{j} \in \mathbb{R}^{q}, j \in J:=\{1, \ldots, n\}$, and assume $0<f_{i}<1, i \in Q:=$ $\{1, \ldots, q\}$. This assumption can be made without loss of generality since setting $x_{i}^{\prime}=x_{i}-\left\lfloor f_{i}\right\rfloor$ and $f_{i}^{\prime}=f_{i}-\left\lfloor f_{i}\right\rfloor, i \in Q$, we have that $x_{i}^{\prime}, f_{i}^{\prime}, i \in Q$ satisfy the assumption. The set

$$
P_{L}:=\left\{(x, s) \in \mathbb{R}^{q} \times \mathbb{R}^{n}:(x, s) \text { satisfies }(2.1)\right\}
$$

is the polyhedral cone with apex at $(x, s)=(f, 0)$ defined by the constraints that are tight for this particular basic solution. Imposing the integrality constraints on the basic components we get the mixed integer set

$$
P_{I}:=\left\{(x, s) \in P_{L}: x_{i} \text { integer, } i \in Q\right\},
$$

whose convex hull, conv $P_{I}$, is Gomory's corner polyhedron (Gomory 1969), or the integer hull of a MIP over the cone $P_{L}$. The main objective of the papers mentioned 
in the introduction was to study the structure of $P_{I}$ for small $q$, with a view of characterizing the facets of conv $P_{I}$ and minimal valid inequalities for $P_{I}$.

Consider now the following disjunctive relaxation of $P_{I}$, obtained by replacing the integrality constraints on $x_{i}$ with the simple disjunctions $x_{i} \leq 0 \vee x_{i} \geq 1, i \in Q$ :

$$
P_{D}:=\left\{(x, s) \in P_{L}: x_{i} \leq 0 \vee x_{i} \geq 1, i \in Q\right\}
$$

Like $P_{I}, P_{D}$ is a nonconvex set. Its convex hull, conv $P_{D}$, which we call the simple disjunctive hull, is a weaker relaxation of $P_{I}$ than conv $P_{I}$, i.e. conv $P_{D} \supseteq \operatorname{conv} P_{I}$, but it is easier to handle, since it is the convex hull of the union of $2^{q}$ polyhedra. Thus one can apply disjunctive programming and lift-and-project techniques to generate facets of conv $P_{D}$ at a computational cost that for small $q$ seems acceptable. In this context, the crucial question is of course, how much weaker is the relaxation conv $P_{D}$ than conv $P_{I}$ ? We will pose this question in a more specific form that will enable us to give it a practically useful answer: when is it that a facet defining inequality for conv $P_{D}$ is also facet defining for conv $P_{I}$ ? In other words, which facets of the (simple) disjunctive hull are also facets of the integer hull? Before addressing this question, however, we will take a side-step, by introducing a third kind of hull. If we strengthen the disjunctive relaxation of $P_{I}$ by replacing the inequalities in the disjunctions $x_{i} \leq 0 \vee x_{i} \geq 1, i \in Q$, with equations, we get the set

$$
P_{D}^{=}:=\left\{(x, s) \in P_{L}: x_{i}=0 \vee x_{i}=1, i \in Q\right\},
$$

whose convex hull, conv $P_{D}^{=}$, we call the $0-1$ disjunctive hull. For a general mixed integer program, the $0-1$ disjunctive hull is not a valid relaxation, in that it may cut off nonbinary feasible integer points. Indeed, we have

$$
\operatorname{conv} P_{D} \supseteq \operatorname{conv} P_{I} \supseteq \operatorname{conv} P_{D}^{=},
$$

where both inclusions are strict and are valid in the context of mixed integer 0-1 programs only, since all the non-0-1 integer points that it cuts off are infeasible. Hence conv $P_{D}^{=}$is equivalent to the convex hull of $P_{I} \cap\left\{x: x_{i} \leq 1, i \in Q\right\}$, or the integer hull of $P_{I}$ reinforced with the bounds on the $x_{i}$. However, as we will see later on, generating facets of conv $P_{D}^{=}$requires roughly the same computational effort as generating facets of conv $P_{D}$.

The upshot of this is that for the important class of mixed integer 0-1 programs, all facet defining inequalities of conv $P_{D}^{=}$are facet defining for the integer hull $P_{I}$. Furthermore, from the sequential convexification theorem of disjunctive programming, all such inequalities are of split rank $\leq q$, i.e. they can be obtained by applying a split cut generating procedure at most $q$ times recursively.

The set $P_{D}$ of (2.4) is the collection of those points $(x, s) \in \mathbb{R}^{q} \times \mathbb{R}^{n}$ satisfying (2.1) and $x_{i} \leq 0 \vee x_{i} \geq 1, i \in Q$. Put in disjunctive normal form, this last constraint set becomes 
Fig. 2 Orthant-cones

for the case $q=2$
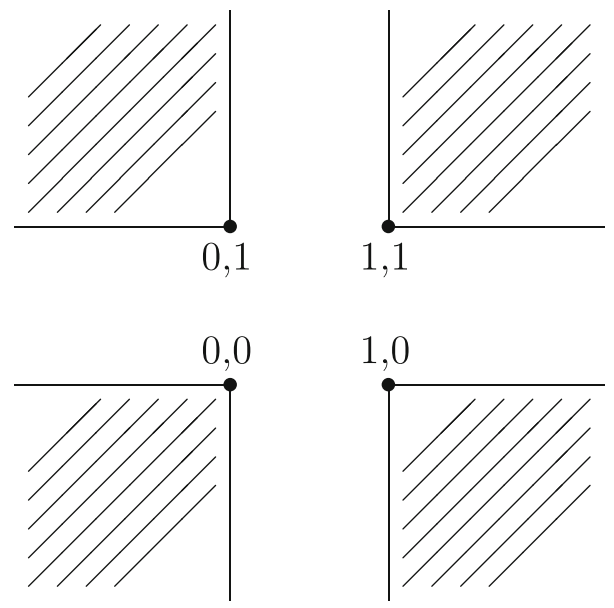

$$
\left(\begin{array}{c}
x_{1} \leq 0 \\
x_{2} \leq 0 \\
\vdots \\
x_{q} \leq 0
\end{array}\right) \vee\left(\begin{array}{c}
x_{1} \geq 1 \\
x_{2} \leq 0 \\
\vdots \\
x_{q} \leq 0
\end{array}\right) \vee \cdots \vee\left(\begin{array}{c}
x_{1} \geq 1 \\
x_{2} \geq 1 \\
\vdots \\
x_{q} \geq 1
\end{array}\right)
$$

Each term of (2.6) defines an orthant-cone with apex at a vertex of the $q$-dimensional unit cube. These $2^{q}$ orthant-cones are illustrated for $q=2$ in Fig. 2.

Using (2.1) to eliminate the $x$-components and denoting by $r^{i}$ the $i$ th row of the $q \times n$ matrix $R=\left(r^{j}\right)_{j=1}^{n},(2.6)$ can be represented in $\mathbb{R}^{n}$ as $s \geq 0$ and

$$
\left(\begin{array}{c}
-r^{1} s \geq f_{1} \\
-r^{2} s \geq f_{2} \\
\vdots \\
-r^{q} s \geq f_{q}
\end{array}\right) \vee\left(\begin{array}{c}
r^{1} s \geq 1-f_{1} \\
-r^{2} s \geq f_{2} \\
\vdots \\
-r^{q} s \geq f_{q}
\end{array}\right) \vee \cdots \vee\left(\begin{array}{c}
r^{1} s \geq 1-f_{1} \\
r^{2} s \geq 1-f_{2} \\
\vdots \\
r^{q} s \geq 1-f_{q}
\end{array}\right)
$$

If $P_{i}^{(n)} \subseteq \mathbb{R}^{n}$ denotes the polyhedron defined by the $i$ th term of this disjunction plus the constraints $s \geq 0$, then $P_{D}$ can be defined in $n$-space as $P_{D}^{(n)}=\cup_{i=1}^{t} P_{i}^{(n)}$ where $t=2^{q}$. Furthermore, we have the following:

Theorem 2.1 conv $P_{D}^{(n)}$ is the set of those $s \in \mathbb{R}^{n}$ satisfying $s \geq 0$ and all the inequalities $\alpha s \geq 1$ whose coefficient vectors $\alpha \in \mathbb{R}^{n}$ satisfy the system 


$$
\begin{aligned}
& \alpha+r^{1} u_{11}+\cdots+r^{q} u_{1 q} \quad \geq 0 \\
& \alpha \quad-r^{1} u_{21}+\cdots+r^{q} u_{2 q} \quad \geq 0 \\
& \alpha \\
& \begin{array}{rlrl}
f_{1} u_{11}+\cdots+f_{q} u_{1 q} & \geq 1 \\
\left(1-f_{1}\right) u_{21}+\cdots+f_{q} u_{2 q} & & \geq 1
\end{array} \\
& -r^{1} u_{t 1}-\cdots-r^{q} u_{t q} \geq 0 \\
& \left(1-f_{1}\right) u_{t 1}+\cdots+\left(1-f_{q}\right) u_{t q} \geq 1
\end{aligned}
$$

for some $u_{i k} \geq 0, i=1, \ldots, t=2^{q}, k=1, \ldots, q$.

Proof Applying the basic theorem of Disjunctive Programming to conv $P_{D}^{(n)}$ we introduce auxiliary variables $s^{i} \in \mathbb{R}^{n}, z_{i} \in \mathbb{R}, i=1, \ldots, t=2^{q}$, and obtain the higherdimensional representation

$$
\begin{aligned}
& s-s^{1}-s^{2} \ldots-s^{t} \\
& -r^{1} s^{1}-f_{1} z_{1} \\
& -r^{2} s^{1} \\
& -f_{2} z_{1} \\
& =0 \\
& \geq 0 \\
& \geq 0 \\
& -r^{q} s^{1} \\
& -f_{q} z_{1} \\
& -\left(1-f_{1}\right) z_{2} \\
& -f_{2} z_{2} \\
& \geq 0 \\
& r^{1} s^{2} \\
& -r^{2} s^{2} \\
& \text { : } \\
& -r^{q} s^{2} \\
& -f_{q} z_{2} \\
& \geq 0 \\
& \geq 0 \\
& \text { : } \\
& \geq 0 \\
& -r^{1} s^{t} \\
& -r^{2} s^{t} \\
& -\left(1-f_{1}\right) z_{t} \geq 0 \\
& -\left(1-f_{2}\right) z_{t} \geq 0 \\
& -r^{q} s^{t} \\
& -\left(1-f_{q}\right) z_{t} \geq 0 \\
& z_{1} \\
& +z_{2}+\cdots \\
& +z_{t}=1 \\
& s^{i} \geq 0, i=1, \ldots, t ; \quad z_{i} \geq 0, i=1, \ldots, t .
\end{aligned}
$$

Projecting this system onto the $s$-space with multipliers $\alpha ; u_{11}, \ldots, u_{1 q} ; u_{21}, \ldots$, $u_{2 q} ; \ldots ; u_{t 1}, \ldots, u_{t q}$, we obtain

$$
\begin{aligned}
& \alpha+r^{1} u_{11} \quad+\cdots+r^{q} u_{1 q} \\
& \geq 0 \\
& \alpha \\
& -\beta+f_{1} u_{11}+\cdots+f_{q} u_{1 q} \\
& -r^{1} u_{t 1} \quad-\cdots-\quad-r^{q} u_{t q} \geq 0 \\
& \vdots \\
& -\beta \\
& \geq 0
\end{aligned}
$$

As the system (2.10) is homogeneous, it can be normalized by truncating the cone that it represents. Applying the normalization $\beta=1$ (clearly $\beta=-1$ does not yield 
any cuts since it makes (2.10) unbounded) we obtain the representation given in the theorem.

In order to restate the system (2.8) in a more concise form, for each $i \in\{1, \ldots, t\}$ we partition the index set $Q:=\{1, \ldots, q\}$ into $Q_{i}^{+}:=\left\{k \in Q: u_{i k}\right.$ has coefficient vector $\left.r^{k}\right\}$ $Q_{i}^{-}:=\left\{k \in Q: u_{i k}\right.$ has coefficient vector $\left.-r^{k}\right\}$, with $Q_{i}^{+} \cup Q_{i}^{-}=Q, i=1, \ldots, t=2^{k}$. Then (2.8) can be restated as

$$
\begin{gathered}
\alpha+\sum\left(r^{k} u_{i k}: k \in Q_{i}^{+}\right)-\sum\left(r^{k} u_{i k}: k \in Q_{i}^{-}\right) \quad \geq 0, i=1, \ldots, t \\
\sum\left(f_{k} u_{i k}: k \in Q_{i}^{+}\right)+\sum\left(\left(1-f_{k}\right) u_{i k}: k \in Q_{i}^{-}\right) \geq 1, i=1, \ldots, t \\
u_{i k} \geq 0, i=1, \ldots, t=2^{q}, k=1, \ldots, q
\end{gathered}
$$

The system (2.8') has several interesting properties described in the next few propositions.

Proposition 2.2 For any $p \in \mathbb{R}^{n}, p>0$, all optimal basic solutions to the cut generating linear program

$$
\min \left\{p \alpha:(\alpha, u) \text { satisfies }\left(2.8^{\prime}\right)\right\}
$$

are of the form

$$
\alpha_{j}=\max \left\{\alpha_{j}^{1}, \ldots, \alpha_{j}^{t}\right\}
$$

where

$$
\alpha_{j}^{i}:=-\sum\left(r_{j}^{k} u_{i k}: k \in Q_{i}^{+}\right)+\sum\left(r_{j}^{k} u_{i k}: k \in Q_{i}^{-}\right),
$$

$i=1, \ldots, t=2^{q}$, with the $u_{i k}$ satisfying (2.8').

Proof The constraints of (2.8') require

$$
\alpha_{j} \geq \alpha_{j}^{i}, \quad i=1, \ldots, t, j=1, \ldots, n .
$$

Suppose there is an optimal solution to $(\mathrm{CGLP})_{Q}$ such that $\alpha_{j_{*}}>\max \left\{\alpha_{j_{*}}^{i}: i=\right.$ $1, \ldots, t\}$ for some $j_{*} \in\{1, \ldots, n\}$. Then setting $\alpha_{j_{*}}$ equal to the maximum on the right-hand side, and leaving $\alpha_{j}$ unchanged for all $j \neq j_{*}$ yields a better solution, contrary to the assumption.

Proposition 2.3 In any valid inequality $\alpha s \geq 1$ for conv $P_{D}^{(n)}, \alpha_{j} \geq 0, j=1, \ldots, n$.

Proof From (2.11), $\alpha_{j} \geq \alpha_{j}^{i}$ for all $i=1, \ldots, 2^{q}$, and in view of the presence of all sign patterns of $r_{j}^{k} u_{i k}$ in the expressions (2.12), there is always an index $i \in\left\{i, \ldots, 2^{q}\right\}$ with $\alpha_{j}^{i} \geq 0$. 
Proposition 2.4 For any basic solution $(\alpha, u)$ to $(C G L P)_{Q}$ that satisfies as strict inequality some of the nonhomogeneous constraints of $\left(2.8^{\prime}\right)$, there exists a basic solution $(\bar{\alpha}, \bar{u})$, with $\bar{\alpha}=\alpha$, that satisfies at equality all the nonhomogeneous constraints of $(C G L P)_{Q}$.

Proof Let $(\alpha, u)$ be a basic solution to $(\mathrm{CGLP})_{Q}$ that satisfies as strict inequality some of the nonhomogeneous constraints of (2.8'). W.l.o.g., assume that

$$
f_{1} u_{11}+\cdots+f_{q} u_{1 q}-\theta=1
$$

is one of those constraints with the surplus variable $\theta$ positive in the solution $(\alpha, u)$. We will show that there exists a solution $(\bar{\alpha}, \bar{u})$, with $\bar{\alpha}=\alpha$ and $\bar{u}_{i k}=u_{i k}$ for all $i \neq 1$ and all $k$, such that

$$
f_{1} \bar{u}_{11}+\cdots+f_{q} \bar{u}_{1 q}=1
$$

Applying this argument recursively then proves the Proposition.

Fix all variables of $(\mathrm{CGLP})_{Q}$ except for $u_{11}, \ldots, u_{1 q}$, at their values in the current solution. The fixing includes all the surplus variables except those in the $n+1$ rows containing $u_{11}, \ldots, u_{1 q}$. This leaves the following constraint set in the free variables:

$$
\begin{aligned}
& -r_{j}^{1} u_{11}-\cdots-r_{j}^{q} u_{1 q}+t_{j}=\bar{\alpha}_{j} \quad j=1, \ldots, n \\
& f_{1} u_{11}+\cdots+f_{q} u_{1 q}-\theta=1 \\
& u_{11}, \ldots, u_{1 q} \geq 0, t_{j} \geq 0, j=1, \ldots, n, \theta \geq 0 .
\end{aligned}
$$

Here $\theta, t_{j}$ represent the surplus variables of the respective constraints. We claim that this system has a solution with $\theta=0$. To see this, consider the linear program

$$
\min \left\{\theta: u_{i k}, t_{j} \text { and } \theta \text { satisfy (2.13) }\right\}
$$

and its dual,

$$
\max \lambda_{0}+\sum_{j=1}^{n} \bar{\alpha}_{j} \lambda_{j}
$$

subject to

$$
\begin{aligned}
f_{1} \lambda_{0}-\sum_{j=1}^{n} r_{j}^{1} \lambda_{j} & \leq 0 \\
\vdots & \vdots \\
f_{q} \lambda_{0}-\sum_{j=1}^{n} r_{j}^{q} \lambda_{j} & \leq 0 \\
-\lambda_{0} & \leq 1 \\
\lambda_{j} & \leq 0, j=1, \ldots, n .
\end{aligned}
$$


Since $\bar{\alpha}_{j} \geq 0, j=1, \ldots, n$, it is not hard to see that the dual linear program has an optimal solution $\lambda_{0}=0, \lambda_{j}=0, j=1, \ldots, n$ and hence the primal has an optimal solution with $\theta=0$.

The obvious and important consequence of Proposition 2.4 is that for all practical purposes we can replace all $2^{q}$ nonhomogeneous inequalities in the constraint set $\left(2.8^{\prime}\right)$ of $(\mathrm{CGLP})_{Q}$ with equations. In view of Proposition 2.2, it then follows that we may restrict our attention to basic feasible solutions that satisfy at equality $n+2^{q}$ out of the $n \times 2^{q}+2^{q}$ inequalities of $\left(2.8^{\prime}\right)$ other than the nonnegativity constraints.

At this point we introduce the characterization of conv $P_{D}^{=}$, the $0-1$ disjunctive hull defined by (2.5), closely related to that of conv $P_{D}$. Just as in the case of $P_{D}$, we denote by $P_{D}^{=(n)}$ the union of polyhedra in $\mathbb{R}^{n}$ representing the disjunction (2.7) in which all the inequalities have been replaced by equations. The following Theorem is the analog of Theorem 2.1 for this case.

Theorem 2.5 conv $P_{D}^{=(n)}$ is the set of those $s \in \mathbb{R}^{n}$ satisfying $s \geq 0$ and all inequalities $\alpha s \geq \beta$ whose coefficients satisfy the system

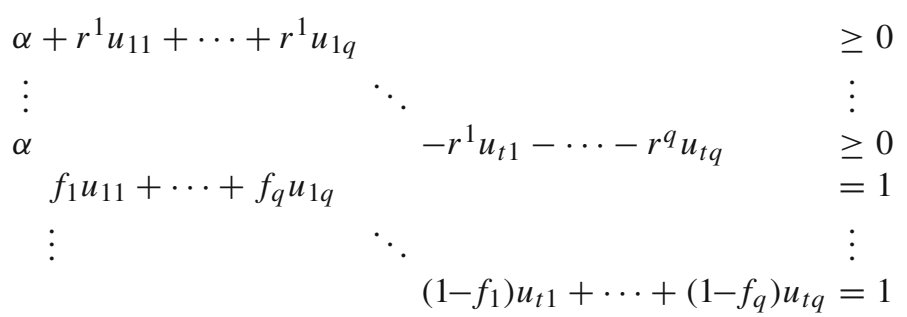

for some $u_{i k}, i=1, \ldots, t=2^{q}, k=1, \ldots, q$.

Proof The proof of Theorem 2.1 goes through with the following modifications. Since the inequalities in the disjunction (2.7) are all replaced with equations, the inequalities in the system (2.9), other than the nonnegativity constraints, also become equations. As a consequence, the variables $u_{i k}$ of the projected system (2.10) become unrestricted in sign. The remaining difference between (2.14) and (2.8) is the fact that in (2.14) the last $2^{q}$ constraints are equations rather than inequalities. This is due to the fact that Proposition 2.4 applies here too. In other words, if we denote by $\left(2.14^{\prime \prime}\right)$ the system obtained from (2.14) by replacing the equations containing $\beta$ with inequalities $\geq$, then for any basic solution $(\alpha, u)$ to $(\mathrm{CGLP})_{Q}$ that satisfies as strict inequalities some of the constraints $\left(2.14^{\prime \prime}\right)$ containing $\beta$, there exists a basic solution $(\bar{\alpha}, u)$, with $\bar{\alpha}=\alpha$, that satisfies at equality all the constraints containing $\beta$. The proof is essentially the same as that of Proposition 2.4.

Thus the only difference between the systems describing conv $P_{D}^{(n)}$ and conv $P_{D}^{=(n)}$ is that the variables $u_{i k}$ and hence the coefficients $\alpha_{j}$ of the latter can be of any sign.

We now return to the simple disjunctive hull, conv $P_{D}$, and describe its vertices.

Remark 2.6 Every vertex of conv $P_{D}^{(n)}$ is a vertex of some $P_{i}^{(n)}, i \in\left\{1, \ldots, 2^{q}\right\}$. 
Next we describe the vertices of $P_{i}^{(n)}, i \in\left\{1, \ldots, 2^{q}\right\}$. We will call a vertex of conv $P_{D}^{(n)}$ (of $P_{i}^{(n)}$ ) integer if it defines an integer $x$ through (2.1); in other words if $f_{i}+r^{i} s$ is integer for $i=1, \ldots, q$. All other vertices will be called fractional.

For any particular $i_{*} \in\left\{1, \ldots, 2^{q}\right\}$,

$$
P_{i_{*}}^{(n)}:=\left\{s \in \mathbb{R}_{+}^{n}: r_{h} s \leq-f_{h}, h \in Q_{i_{*}}^{+}, r_{h} s \geq 1-f_{h}, h \in Q_{i_{*}}^{-}\right\}
$$

where $\left(Q_{i_{*}}^{+}, Q_{i_{*}}^{-}\right)$is the partition of $Q$ for $i_{*}$, used in (2.8').

Proposition $2.7 P_{i_{*}}^{(n)}$ can have three kinds of vertices, distinguished by the corresponding $x$-vectors that belong to one of these types:

(a) 0-1 vertices: $x_{h}=0, h \in Q_{i_{*}}^{+}$and $x_{h}=1, h \in Q_{i_{*}}^{-}$.

(b) non-binary integer vertices: $x_{h} \in \mathbb{Z}, h \in Q, x_{h} \leq 0, h \in Q_{i_{*}}^{+}, x_{h} \geq 1, h \in$ $Q_{i_{*}}^{-}$, with at least one inequality strict.

(c) fractional vertices: $x_{h} \leq 0, h \in Q_{i_{*}}^{+}, x_{h} \geq 1, h \in Q_{i_{*}}^{-}$, with at least one component of $x$ fractional.

Proof The three cases become exhaustive if the following fourth one is added: (d) fractional vertices with $0<x_{h}<1$ for some $h \in Q$. But this case clearly violates at least one of the constraints defining $P_{i_{*}}^{(n)}$.

Note that $P_{i_{*}}^{(n)}$ can have several distinct vertices with the same associated $x$-vector, corresponding to basic solutions with the same $x$-component. Note also that if a component $x_{h}$ of a vertex is fractional, then $x_{h}<0$ or $x_{h}>1$.

The next theorem characterizes the facets of the simple disjunctive hull.

Theorem 2.8 The inequality $\bar{\alpha} s \geq 1$ defines a facet of conv $P_{D}^{(n)}$ if and only if there exists an objective function of the linear program $(C G L P)_{Q}$ of Proposition 2.2 with $p>0$ such that all optimal solutions $(\alpha, u)$ have $\alpha=\bar{\alpha}$.

Proof outline. This is a special case of Theorem 4.6 of Balas (1998). The inequality $\bar{\alpha} x \geq 1$ defines a facet of conv $P_{D}^{(n)}$ if and only if $\bar{\alpha}$ is a vertex of the polar of conv $P_{D}^{(n)}$, which is the projection of (2.8) onto the $\alpha$-space. But $\bar{\alpha}$ is a vertex of this polar if and only if there exists an objective function vector $p>0$ such that $p \alpha$ attains its unique minimum at $\bar{\alpha}$.

If the system (2.4) defining $P_{L}$ is of full row rank $q$, then the dimension of conv $P_{D}$ is $n$, since there are $q+n$ variables and $q$ independent equations. The dimension of conv $P_{D}^{(n)}$ is also $n$, so the facets of conv $P_{D}^{(n)}$ are of dimension $n-1$.

From a computational standpoint, the most important feature of (CGLP) $)_{Q}$ is that the facets of the $n$-dimensional conv $P_{D}^{(n)}$ can be generated by solving a smaller CGLP in a subspace of at most $t=2^{q}$ variables $\alpha_{j}$, and lifting the resulting inequality into the full space.

Since our cuts are derived from a disjunction with $2^{q}$ terms, if we want to create a subproblem in which all terms are represented, we need $2^{q}$ out of the $n$ variables $\alpha_{j}$ of our $(\mathrm{CGLP})_{Q}$. Furthermore, the $2^{q}$ vectors $r^{j}$ corresponding to these $\alpha_{j}$ have to span the subspace $\mathbb{R}^{q}$ of the $x$-variables. Solving the (CGLP) $Q$ in this subspace yields $2^{q}$ 
values $\alpha_{j}$ and $q \times 2^{q}$ associated multipliers $u_{i k}, i=1, \ldots, 2^{q}, k=1, \ldots, q$; and these multipliers can then be used to compute the remaining components of $\alpha$, given by the expressions (2.11) and (2.12). The significance of this is that the computational cost of generating facets of conv $P_{D}$ grows only linearly with $n$. Of course this cost grows exponentially with $q$, but the approach discussed here is being considered for small $q$.

The idea of generating cuts in a subspace of the original higher dimensional cut generating linear program and then lifting them to the full space goes back to Balas et al. (1993), Balas and Perregaard (2003), where lift-and-project cuts were generated from a two-term disjunction by working in the subspace of the fractional variables of the LP solution. Here we are working with a $2^{q}$-term disjunction, and are considering a different subspace, suggested by the structure of the problem at hand, but the lifting procedure is essentially the same as the one used in Balas et al. $(1993,1996)$.

The choice of the $2^{q}$ vectors $r^{j}$ for the problem in the subspace is intimately related to the question of deciding which facets of the disjunctive hull are also facets of the integer hull, as it will be seen in Sec. 4. The best way to address this question and that of the subspace to be chosen is to first interpret the inequalities defining the disjunctive hull as intersection cuts.

\section{Geometric interpretation: cuts from the $q$-dimensional parametric cross-polytope}

Consider the $q$-dimensional unit cube centered at $(0, \ldots, 0), K_{q}:=\left\{x \in \mathbb{R}^{q}:-\frac{1}{2} \leq\right.$ $\left.x_{j} \leq \frac{1}{2}, j \in Q\right\}$. Its polar, $K_{q}^{o}:=\left\{x \in \mathbb{R}^{q}: x y \leq 1, \forall y \in K\right\}$, is known to be the $q$-dimensional octahedron or cross-polytope; which, when scaled so as to circumscribe the unit cube, is the outer polar of $K_{q}$ :

$$
K_{q}^{*}=\left\{x \in \mathbb{R}^{q}:|x| \leq \frac{1}{2} q\right\},
$$

where $|x|=\sum\left(\left|x_{j}\right|: j=1, \ldots, q\right)$. Equivalently, $|x| \leq \frac{1}{2} q$ can be written as the system

$$
\begin{aligned}
-x_{1}-\cdots-x_{q} & \leq \frac{1}{2} q \\
x_{1}-\cdots-x_{q} & \leq \frac{1}{2} q \\
& \vdots \\
x_{1}+\cdots+x_{q} & \leq \frac{1}{2} q
\end{aligned}
$$

of $t=2^{q}$ inequalities in $q$ variables.

Moving the center of the coordinate system to $\left(\frac{1}{2}, \cdots, \frac{1}{2}\right)$ changes the right-hand side coefficient of the $i$ th inequality in (3.1) from $\frac{1}{2} q$ to a value equal to the sum of positive coefficients on the left-hand side of the inequality. Indeed, if $q^{+}$and $q^{-}$ denote the number of positive and negative coefficients, then $\frac{1}{2} q+\frac{1}{2} q^{+}-\frac{1}{2} q^{-}=q^{+}$.

Next we introduce the parameters $v_{i k}, i=1, \ldots, t=2^{q}, k=1, \ldots, q$, to obtain the system 


$$
\begin{gathered}
-v_{11} x_{1}-\cdots-v_{1 q} x_{q} \leq 0 \\
v_{21} x_{1}-\cdots-v_{2 q} x_{q} \leq v_{21} \\
-v_{31} x_{1}+\cdots-v_{3 q} x_{q} \leq v_{31} \\
\vdots \\
\vdots \\
v_{t 1} x_{1}+\cdots+v_{t q} x_{q} \leq v_{t 1}+\ldots+v_{t q} \\
v_{i k} \geq 0, i=1, \ldots, t=2^{q}, k=1, \ldots, q .
\end{gathered}
$$

Note that the constraints of (3.2) are of the form

$$
\sum_{k \in \widetilde{Q}_{i}^{+}} v_{i k} x_{k}-\sum_{k \in \widetilde{Q}_{i}^{-}} v_{i k} x_{k} \leq \sum_{k \in \widetilde{Q}_{i}^{+}} v_{i k},
$$

where $\widetilde{Q}_{i}^{+}$and $\widetilde{Q}_{i}^{-}$are the sets of indices for which the coefficient of $x_{k}$ is $+v_{i k}$ and $-v_{i k}$, respectively. Note also that all inequalities that have the same number of coefficients with the plus sign have the same right-hand side, equal to the sum of these coefficients.

The system (3.2) is homogeneous in the parameters $v_{i k}$, so every one of its inequalities can be normalized. Since we are looking for a connection with the system (2.8) defining $(\mathrm{CGLP})_{Q}$, we will use the normalization given by this system and Proposition 2.4 , i.e.

$$
\begin{array}{rlrl}
f_{1} v_{11}+\cdots+ & f_{q} v_{1 q} & =1 \\
\left(1-f_{1}\right) v_{21}+\cdots+ & f_{q} v_{2 q} & =1 \\
\cdots & \cdots & \\
\left(1-f_{1}\right) v_{t 1}+\cdots+\left(1-f_{q}\right) v_{t q} & =1 .
\end{array}
$$

Note that these normalization constraints are of the general form

$$
\sum_{h \in \widetilde{Q}_{i}^{+}}\left(1-f_{k}\right) v_{i k}+\sum_{h \in \widetilde{Q}_{i}^{-}} f_{k} v_{i k}=1 .
$$

Let $\widetilde{K}^{*}(v)$ denote the parametric cross-polytope defined by (3.2) and (3.3). It is not hard to see that for any fixed set of $v_{i k}$, (3.2) defines a convex polyhedron in $x$-space that contains in its boundary all $x \in \mathbb{R}^{q}$ such that $x_{k} \in\{0,1\}, k \in Q$, hence is suitable for generating intersection cuts. Furthermore, letting $\widetilde{K}^{*(n)}(v)$ be the expression for $\widetilde{K}^{*}(v)$ in the space of the $s$-variables, obtained by substituting $f+R s$ for $x$ into (3.2), we have

Theorem 3.1 For any values of the parameters $v_{i k}$ satisfying (3.2) and (3.3), the intersection cut $\widetilde{\alpha} s \geq 1$ from $\widetilde{K}^{*(n)}(v)$ has coefficients $\widetilde{\alpha}_{j}=\frac{1}{s_{j}^{*}}$, where

$$
s_{j}^{*}=\max \left\{s_{j}: f+r^{j} s_{j} \in K^{*(n)}(v)\right\} .
$$

Proof This is a special case of Theorem 1.1. 
In order to compare the intersection cut $\widetilde{\alpha} s \geq 1$ with the cut $\alpha s \geq 1$ from the $q$-term disjunction (2.7), we have to restate (3.4) in terms of the system of inequalities defining $\widetilde{K}^{*(n)}(v)$. This means that $f+r^{j} s_{j}^{*}$ has to be expressed as the intersection point of the ray $f+r^{j} s_{j}, s_{j} \geq 0$, with the first facet of $K^{*(n)}(v)$ encountered. This yields

$$
s_{j}^{*}=\min \left\{s_{j}^{1}, \ldots, s_{j}^{t}\right\}
$$

where the $s_{j}^{i}$ are obtained by substituting $f_{k}+\sum_{h=1}^{n} r_{j}^{k} s_{h}$ for $x_{k}, k=1, \ldots, q$ into the $i$ th inequality of (3.2), and setting $s_{h}=0$ for all $h \neq j$ :

$$
\begin{aligned}
& s_{j}^{i}=\max \left\{s_{j}:\left(\sum_{k \in \widetilde{Q}_{i}^{+}} v_{i k} r_{j}^{k}-\sum_{k \in \widetilde{Q}_{i}^{-}} v_{i k} r_{j}^{k}\right) s_{j} \leq \sum_{k \in \widetilde{Q}_{i}^{+}} v_{i k}\left(1-f_{k}\right)+\sum_{k \in \widetilde{Q}_{i}^{-}} v_{i k} f_{k}\right\}, \\
& i=1, \ldots, t=2^{q} .
\end{aligned}
$$

Clearly, this maximum is bounded whenever the coefficient of $s_{j}$ is positive, in which case, if we normalize by setting $\sum_{k \in \widetilde{Q}_{i}^{+}} v_{i k}\left(1-f_{k}\right)+\sum_{k \in \widetilde{Q}_{i}^{-}} v_{i k} f_{k}=1$, we obtain

$$
s_{j}^{i}=\left(\sum_{k \in \widetilde{Q}_{i}^{+}} v_{i k} r_{j}^{k}-\sum_{k \in \widetilde{Q}_{i}^{-}} v_{i k} r_{j}^{k}\right)^{-1} .
$$

Comparing (3.5) and (3.6) to the expressions (2.11) and (2.12) for the coefficient $\alpha_{j}$ of the lift-and-project cut $\alpha s \geq 1$ of Proposition 2.2, we find that setting $v_{i k}=u_{i k}$ for all $i, k$, as well as $\widetilde{Q}_{i}^{+}=Q_{i}^{-}$and $\widetilde{Q}_{i}^{-}=Q_{i}^{+}$, we obtain $\widetilde{\alpha}_{j}=\alpha_{j}$.

This proves

Corollary 3.2 The intersection cut $\widetilde{\alpha} s \geq 1$ from the parametric octahedron $\widetilde{K}^{*(n)}(v)$ is the same as the lift-and-project cut $\alpha s \geq 1$ corresponding to the $(C G L P)_{Q}$ solution $(\alpha, u)$, with $v_{i k}=u_{i k}, i=1, \ldots, t, k=1, \ldots, q$.

Given these two derivations of the same cut, which one should we use? Either one or both, depending on the situation: one of them shows how the different parameter vectors $v$ give rise to different incarnations of the parametric cross-polytope $\widetilde{K}^{*(n)}(v)$, with different characteristics of the resulting cuts; the other one allows us to choose the parameter vector either by directly specifying a basis of $(\mathrm{CGLP})_{Q}$, or by optimizing an objective function to find one, thereby determining the shape of the resulting cut.

\section{Facets of the disjunctive hull and the integer hull}

Consider again the disjunctive relaxation of $P_{I}$

$$
P_{D}=\left\{(x, s) \in \mathbb{R}^{q} \times \mathbb{R}^{n}: x=f+R s, s \geq 0, x_{i} \leq 0 \vee x_{i} \geq 1, i \in Q\right\}
$$


introduced at the beginning of Sec. 2, where $x, f \in \mathbb{R}^{q}, R \in \mathbb{R}^{q \times n}$, and $Q:=$ $\{1, \ldots, q\}$. For $i=1, \ldots, t=2^{q}$, let $p^{i}$ be the vertex of $K_{q}$, the $q$-dimensional unit cube, defined by $p_{k}^{i}=0, i \in Q_{i}^{+}, p_{k}^{i}=1, i \in Q_{i}^{-}$.

Next we give a sufficient condition for an inequality $\alpha s \geq 1$ valid for $P_{D}$ to define a facet of conv $P_{I}$, which for small $q$ leads to an efficient procedure for generating inequalities that are facet defining for conv $P_{I}$.

The dimension of $P_{I}^{(n)}$ being $n \geq 2^{q}, \alpha s \geq 1$ defines a facet of conv $P_{I}^{(n)}$ if there exists a subspace $\mathbb{R}^{2^{q}}$ of $\mathbb{R}^{n}$ such that the restriction of $\alpha s \geq 1$ to this subspace defines a facet of conv $P_{I}^{\left(2^{q}\right)}$. If this is the case, then the inequality in question can be lifted to the full space to yield a facet of conv $P_{I}^{(n)}$ by using the $u$-components of the solution $(\alpha, u)$ to the CGLP in the subspace to compute the missing coefficients $\alpha_{j}$.

Theorem 4.1 Let $\alpha s \geq 1$ be a valid inequality for $P_{D}$ corresponding to a basic solution $(\alpha, u)$ of $(C G L P)_{Q}$, and let $p^{i}, i=1, \ldots, 2^{q}$, be the vertices of $K_{q}$. Suppose for each $p^{i}, i=1, \ldots, 2^{q}$, there exists a subset $J_{i} \subset J$ containing the indices of $q$ linearly independent rays $r^{j_{1}}, \ldots, r^{j_{q}}$, and a vector $\lambda \in \mathbb{R}_{+}^{q}$, satisfying

$$
p^{i}-f=\sum_{j=j_{1}}^{j_{q}} \frac{1}{\alpha_{j}} r^{j} \lambda_{j}, \quad \sum_{j=j_{1}}^{j_{q}} \lambda_{j}=1 .
$$

Then the inequality $\sum_{j \in J} \alpha_{j} s_{j} \geq 1$ defines a facet of conv $P_{I}^{(|J|)}$, and its lifting based on the u-components of the solution $(\alpha, u)$ defines a facet of conv $P_{I}^{(n)}$.

Proof Suppose the subset of $2^{q}$ rays indexed by $J$ satisfies the requirements of the Theorem. Then for every $i=1, \ldots, 2^{q}$, the vertex $p^{i}$ of $K^{q}$ satisfies

$$
p^{i}=\sum_{j=j_{1}}^{j_{q}}\left(f-\frac{1}{\alpha_{j}} r^{j}\right) \lambda_{j}, \quad \sum_{j=j_{1}}^{j_{q}} \lambda_{j}=1
$$

for some $\lambda_{j} \geq 0, j=j_{1}, \ldots, j_{q}$, i.e. $p^{i}$ can be expressed as a convex combination of the $q$ points $f+\frac{1}{\alpha_{j}} r^{j}, j=j_{1}, \ldots, j_{q}$. But $f+\frac{1}{\alpha_{j}} r^{j}=f+r^{j} s_{j}^{*}$ is the intersection point of the ray $f+r^{j} s_{j}$ with bd $\widetilde{K}_{q}^{*}$, hence each of these points satisfies $\alpha s=1$ and consequently so does $p^{i}$. Since $\sum_{j \in J} \alpha_{j} s_{j} \geq 1$ is satisfied at equality by $2^{q}$ independent integer points of conv $P_{I}^{(|J|)}$, it defines a facet of the latter. Furthermore, lifting the remaining coefficients $\alpha_{j}$ of the inequality by using the $u$-components of $(\alpha, u)$ yields a facet defining inequality for conv $P_{I}^{(n)}$.

The sufficient condition of Theorem 4.1 is not necessary. There are situations in which the condition (4.1) is not satisfied for all $2^{q}$ vertices $p^{i}$ of $K^{q}$, but for some of the vertices (4.1) it is satisfied for more than one set $J_{i} \subset J$ of linearly independent rays. This corresponds to the fact that a set of $2^{q}$ independent integer points of $\operatorname{conv} P_{I}^{(|J|)}$ may contain more than one point with the same $x$-component. Thus, if fewer than $2^{q}$ vertices $p^{i}$ of $K^{q}$ satisfy (4.1), but some of them satisfy (4.1) for more than one set $J_{i}$, 
then it is still possible to have $2^{q}$ independent integer points of conv $P_{I}^{(|J|)}$ satisfying $\sum_{j \in J} \alpha_{j} s_{j}=1$.

So far we have discussed intersection cuts from $q$ rows, i.e. cuts from disjunctions with $2^{q}$ terms, for arbitrary $q>1$. In the next section we illustrate our approach on the case $q=2$, and thereafter we address some related topics also on the case $q=2$. The study of this case is meant to lay the groundwork for a subsequent study of the cases $q>2$.

\section{The two-row case}

We now restrict our attention to the case $q=2$, i.e. we consider two rows from a simplex tableau of a MIP problem with the variables $x_{1}, x_{2}$ and $s_{j}, j \in J$ :

$$
\begin{aligned}
P_{L}=\left\{(x, s) \in \mathbb{R}^{2+|J|}: x_{1}\right. & =f_{1}+\sum_{j \in J} r_{j}^{1} s_{j} \\
x_{2} & =f_{2}+\sum_{j \in J} r_{j}^{2} s_{j} \\
s_{j} & \geq 0 \quad j \in J\} .
\end{aligned}
$$

where $x_{1}, x_{2}$ are basic variables required to be integers and $s_{j}, j \in J$ are non-basic. This is the case studied by Andersen et al. (2007). Let $P_{I}=\left\{(x, s) \in \mathbb{Z}^{2} \times \mathbb{R}^{|J|}\right.$ : $\left.(x, s) \in P_{L}\right\}$, and $0<f_{1}, f_{2}<1$. The column vectors $r_{j}, j \in J$, represent the extreme rays of the cone in $\mathbb{R}^{|J|}$ with apex at $\left(f_{1}, f_{2}\right)$.

We will say that a ray $r_{j}$ in (5.1) hits an orthant-cone $Q_{i}, i \in\{1, \ldots, 4\}$ if there exists $\lambda_{0}>0$ such that $f+\lambda r_{j} \in Q_{i}$ for all $\lambda \geq \lambda_{0}$.

For the case of two rows the disjunction (2.7) becomes

$$
\left(\begin{array}{l}
-r^{1} s \geq f_{1} \\
-r^{2} s \geq f_{2}
\end{array}\right) \vee\left(\begin{array}{l}
r^{1} s \geq 1-f_{1} \\
-r^{2} s \geq f_{2}
\end{array}\right) \vee\left(\begin{array}{l}
r^{1} s \geq 1-f_{1} \\
r^{2} s \geq 1-f_{2}
\end{array}\right) \vee\left(\begin{array}{l}
-r^{1} s \geq f_{1} \\
r^{2} s \geq 1-f_{2}
\end{array}\right)
$$

with $s \geq 0$, and the system (2.8) of Theorem 2.1 becomes

$$
\begin{array}{llll}
\alpha & +r^{1} v_{1} & +r^{2} w_{1} & \geq 0 \\
\alpha-r^{1} v_{2} & +r^{2} w_{2} & \geq 0 \\
\alpha-r^{1} v_{3} & -r^{2} w_{3} & \geq 0 \\
\alpha+r^{1} v_{4} & -r^{2} w_{4} & \geq 0 \\
\quad+f_{1} v_{1} & +f_{2} w_{1} & =1 \\
\quad+\left(1-f_{1}\right) v_{2} & +f_{2} w_{2} & =1 \\
\quad+\left(1-f_{1}\right) v_{3} & +\left(1-f_{2}\right) w_{3} & =1 \\
& +f_{1} v_{4} & +\left(1-f_{2}\right) w_{4} & =1 \\
v_{i}, w_{i} \geq 0 \quad i \in\{1 \ldots 4\} . &
\end{array}
$$


where $v_{i}, w_{i}, i=1, \ldots, 4$ stand for $u_{i 1}, u_{i 2}, i=1, \ldots, t=2^{q}$ (since $q=2, t=$ $\left.2^{q}=4\right)$.

By Proposition 2.2 the cuts generated by the CGLP with constraint set (5.3) and objective function $\min p \alpha$ for some $p>0$ have the form $\alpha s \geq 1$, where

$$
\alpha_{j}=\max \left\{\alpha_{j}^{1}, \alpha_{j}^{2}, \alpha_{j}^{3}, \alpha_{j}^{4}\right\}
$$

with

$$
\begin{array}{ll}
\alpha_{j}^{1}=-r_{j}^{1} \bar{v}_{1} & -r_{j}^{2} \bar{w}_{1} \\
\alpha_{j}^{2}=+r_{j}^{1} \bar{v}_{2} & -r_{j}^{2} \bar{w}_{2} \\
\alpha_{j}^{3}=+r_{j}^{1} \bar{v}_{3} & +r_{j}^{2} \bar{w}_{3} \\
\alpha_{j}^{4}=-r_{j}^{1} \bar{v}_{4} & +r_{j}^{2} \bar{w}_{4} .
\end{array}
$$

Here $\left(\bar{v}_{k}, \bar{w}_{k}\right)$ are the components of the (CGLP) solution.

As discussed in Sec. 3, a cut produced by the CGLP can be viewed as an intersection cut derived from a parametric cross-polytope or octahedron. For given $v, w \geq 0$, we call the polyhedron

$$
\begin{aligned}
P_{\mathrm{octa}}(v, w)=\left\{\left(x_{1}, x_{2}\right) \in \mathbb{R}^{2}:\right. & -v_{1} x_{1}-w_{1} x_{2} \leq 0 \\
& +v_{2} x_{1}-w_{2} x_{2} \leq v_{2} \\
& +v_{3} x_{1}+w_{3} x_{2} \leq v_{3}+w_{3} \\
& \left.-v_{4} x_{1}+w_{4} x_{2} \leq w_{4}\right\}
\end{aligned}
$$

the $(v, w)$-parametric octahedron.

If $v_{i}=0$ or $w_{i}=0$ for some $i \in\{1, \ldots, 4\}$ the $i$ th facet of $P_{\text {octa }}$ is parallel to one of the coordinate axes. If $v_{i}, w_{i}>0$ then the $i$ th facet of $P_{\text {octa }}$ is tilted (note that since we use the normalization $\beta=1, v_{i}$ and $w_{i}$ cannot both be 0 ). Varying the parameters $v, w$, the $(v, w)$-parametric octahedron produces different configurations according to the non-zero components of $v, w$. Depending on the values taken by the parameters, $P_{\text {octa }}(v, w)$ may be a quadrilateral (i.e. a full-fledged octahedron in $\mathbb{R}^{2}$ ), a triangle, or an infinite strip. In the rest of the section we refer to these configurations using the short reference indicated in parenthesis. It can easily be verified that the value-configurations of the parameters $v_{i}, w_{i}$ which give rise to maximal convex sets are the following:

- $(S)$ If exactly four components of $(v, w)$ are positive, $P_{\text {octa }}$ is the vertical strip $\left\{x \in \mathbb{R}^{2}: 0 \leq x_{1} \leq 1\right\}$ if $v_{i}>0, i=1, \ldots, 4$; or the horizontal strip $\left\{x \in \mathbb{R}^{2}\right.$ : $\left.0 \leq x_{2} \leq 1\right\}$ if $w_{i}>0, i=1, \ldots, 4$ (see Fig. 3a, b).

- $\left(T_{A}\right)$ If exactly five components of $(v, w)$ are positive, $P_{\text {octa }}$ is a triangle with one tilted face (type A) (by "tilted" we mean a face that is not parallel to any of the two axes). Figure $3 \mathrm{c}$ illustrates the case with $v_{1}, w_{2}, v_{3}, w_{3}, v_{4}>0 ; w_{1}, v_{2}, w_{4}=0$. When in addition $v_{i}=w_{i}$ for some $i \in\{1, \ldots, 4\} P_{\text {octa }}$ becomes a triangle with vertices $(0,0) ;(2,0) ;(0,2)$ or one of the other three configurations symmetric to this one. This corresponds to what is called a triangle of type 1 in Dey and 
Wolsey (2010). In the general case $T_{A}$ corresponds to a triangle of type 2 in Dey and Wolsey (2010).

- $\left(T_{B}\right)$ If exactly six components of $(v, w)$ are positive, $P_{\text {octa }}$ is a triangle with two tilted faces (type B). Figure $3 \mathrm{~d}$ illustrates the case with $v_{1}, w_{1}, v_{2}, w_{2}, w_{3}, w_{4}>$ $0 ; v_{3}, v_{4}=0$. This configuration corresponds to a triangle of type 2 in Dey and Wolsey (2010).

- $(Q)$ If all eight components of $(v, w)$ are positive, $P_{\text {octa }}$ is a quadrilateral. See Fig. 3e.

The case with seven components of $(v, w)$ positive does not correspond to a maximal parametric octahedron, therefore we do not need to consider it. Suppose all the components are positive except for $v_{1}$ which is 0 . The facet of $P_{\text {octa }}$ corresponding to $(0,0)$ is horizontal and goes through the point $(1,0)$. It is not hard to see that setting $v_{2}=0$ will enlarge the set defined by the parametric octahedron.

For a cut $\sum_{j \in J} \alpha_{j} s_{j} \geq 1$ Andersen et al. (2007) introduce the set

$$
L_{\alpha}=\left\{x \in \mathbb{R}^{2}:(x, s) \in P_{L} \wedge \sum_{j \in J} \alpha_{j} s_{j} \leq 1\right\}
$$

Clearly, $L_{\alpha} \subseteq P_{\text {octa }}(v, w)$, and the inclusion is often strict.

Example Andersen et al. (2007), considered the two rows instance

$$
\begin{aligned}
& x_{1}=\frac{1}{4}+2 s_{1}+1 s_{2}-3 s_{3} \quad+1 s_{5} \\
& x_{2}=\frac{1}{2}+1 s_{1}+1 s_{2}+2 s_{3}-1 s_{4}-2 s_{5}, \\
& x_{1}, x_{2} \in \mathbb{Z}, \quad s \geq 0 .
\end{aligned}
$$

We present the complete description of the disjunctive hull for (5.6). In order to do so we generated the CGLP of (5.6) using the normalization constraint $\beta=1$ and we considered all feasible bases. The CGLP produces five different facets. For each of these we show the configuration of the parametric octahedron that yields the corresponding cut in terms of the $v, w$ variables:

1. Cut $\left(T_{B}\right): 2 s_{1}+2 s_{2}+4 s_{3}+s_{4}+\frac{12}{7} s_{5} \geq 1$

$$
\begin{aligned}
& v_{1}=2 ; v_{2}=\frac{8}{7} ; v_{3}=0 ; v_{4}=0 \\
& w_{1}=1 ; w_{2}=\frac{2}{7} ; w_{3}=2 ; w_{4}=2
\end{aligned}
$$

2. Cut $\left(T_{B}\right): \frac{8}{3} s_{1}+\frac{4}{3} s_{2}+\frac{44}{9} s_{3}+\frac{8}{9} s_{4}+\frac{4}{3} s_{5} \geq 1$

$$
\begin{aligned}
& v_{1}=\frac{20}{9} ; v_{2}=\frac{4}{3} ; v_{3}=\frac{4}{3} ; v_{4}=\frac{4}{9} \\
& w_{1}=\frac{8}{9} ; w_{2}=0 ; w_{3}=0 ; w_{4}=\frac{16}{9}
\end{aligned}
$$



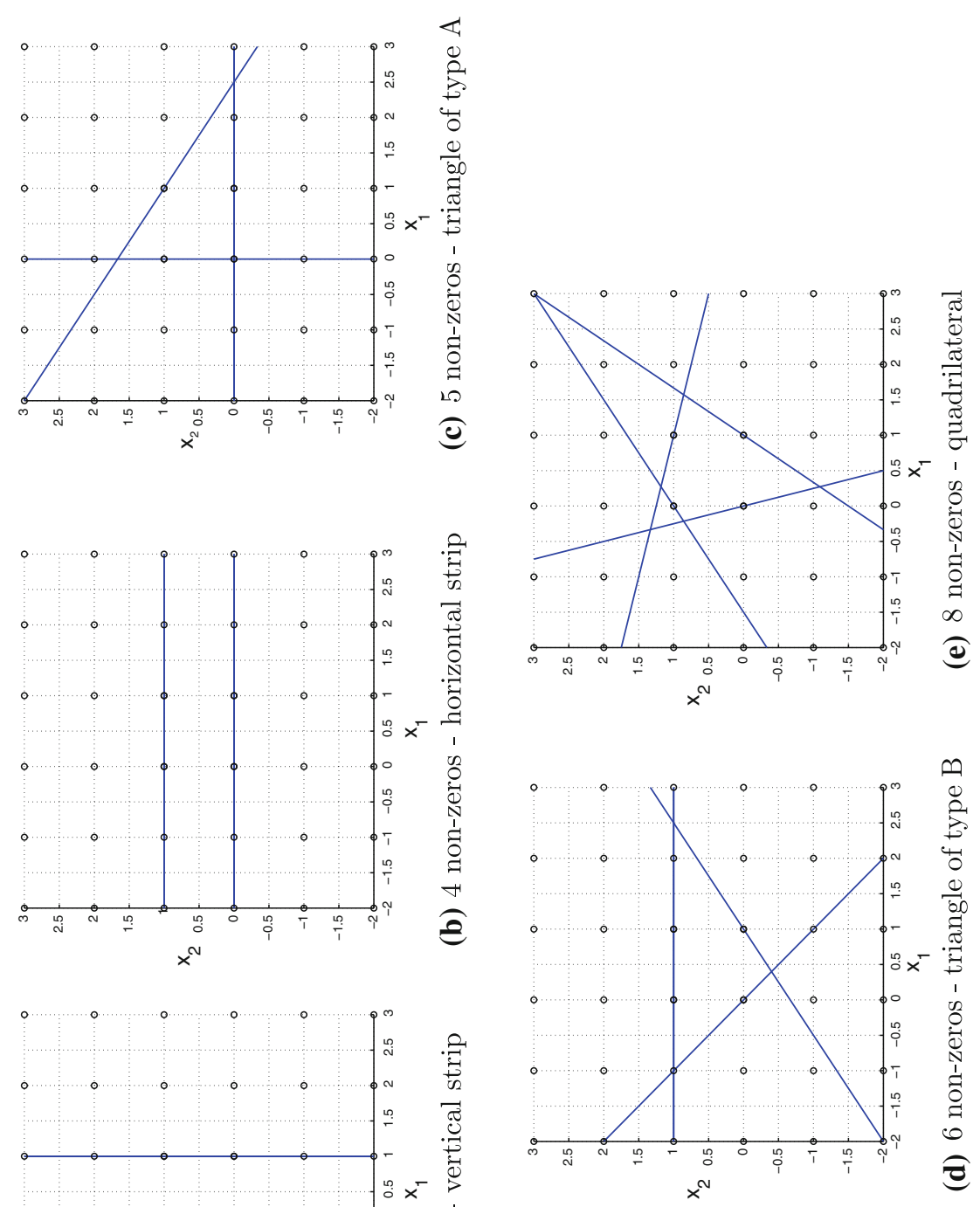

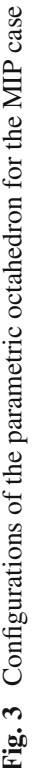


3. $\operatorname{Cut}\left(T_{A}\right): \frac{8}{3} s_{1}+2 s_{2}+4 s_{3}+s_{4}+\frac{4}{3} s_{5} \geq 1$

$v_{1}=2 ; v_{2}=\frac{4}{3} ; v_{3}=0 ; v_{4}=0$

$w_{1}=1 ; w_{2}=0 ; w_{3}=2 ; w_{4}=2$

4. $\operatorname{Cut}(S): \frac{8}{3} s_{1}+\frac{4}{3} s_{2}+12 s_{3}+\frac{4}{3} s_{5} \geq 1$

$v_{1}=4 ; v_{2}=\frac{4}{3} ; v_{3}=\frac{4}{3} ; v_{4}=4$

$w_{1}=0 ; w_{2}=0 ; w_{3}=0 ; w_{4}=0$

5. Cut $\left(T_{B}\right): 2 s_{1}+2 s_{2}+\frac{68}{7} s_{3}+\frac{2}{7} s_{4}+\frac{12}{7} s_{5} \geq 1$

$v_{1}=\frac{24}{7} ; v_{2}=\frac{8}{7} ; v_{3}=0 ; v_{4}=0$

$w_{1}=\frac{2}{7} ; w_{2}=\frac{2}{7} ; w_{3}=2 ; w_{4}=2$.

Of the five facets of $P_{D}$, three are facets for $P_{I}$ : cuts 1,2 and 4 . Note that cut 4 is a split cut and can be derived using only the tableau row corresponding to the variable $x_{2}$. Cut 3 and 5 are facets of $P_{D}$ by Theorem 2.8 .

The condition given in Theorem 4.1 for an inequality $\alpha x \geq 1$, facet defining for the disjunctive hull, to also define a facet of the integer hull specializes for the case $q=2$ to the following. For each of the four vertices $p^{i}$ of $K, p^{i}$ must lie on the line segment between two intersection points of rays $r^{j}$ with the boundary of $P_{\text {octa }}$. As discussed in Sec. 3, the inequalities $\alpha x \geq 1$ can be generated in a subspace of $\leq 2^{q}=4$ variables, and then lifted into the full space by using the multipliers $\left(v_{i}, w_{i}\right), i=1, \ldots, 4$. In Qualizza (2011) two algorithms were implemented for generating facets of the integer hull from $P_{\text {octa }}$, one for the case of a quadrilateral, the other for the case of triangles, both of them linear in $|J|$, the number of rays.

Recently Dash et al. (2010) have generalized the approach of Balas (2009a,b), by considering more general four-term disjunctions that give rise to what they call cross cuts and crooked cross cuts. They relate the closures of their cuts with the split closure and show, among others, that any two-dimensional lattice free cut can be obtained as a crooked cross cut.

\section{Cut strengthening}

Given a valid cut $\alpha s \geq 1$ for the disjunctive hull, if some non-basic variable $s_{j}$ is required to be integral in the original problem formulation, then the cut can be strengthened. There are two main approaches to strengthening cuts for mixed integer programs: one based on the group theoretic approach of Johnson (1974), the other one based on the monoidal cut strengthening of Balas and Jeroslow (1980). 
Both approaches use the integrality constraint on the variables to modularize in one way or another the cut coefficients of such variables. The first approach, adapted to intersection cuts from two rows by Dey and Wolsey (2010), constructs valid lifting functions, like the "trivial" lifting function, using so-called fill-in-procedures. The second approach, as applied to cuts from multiple-term disjunctions, modularizes the maximand in the expression for the cut coefficients by using an appropriate monoid. These two approaches yield outcomes that are sometimes the same, sometimes different. The interpretation mentioned in Dey and Wolsey (2010), that the two are computationally equivalent, only applies to some cases where the monoid used in the second approach is the set of all integer vectors. When a different monoid is used, like in the family of strengthened cuts derived in Sec. 7, the equivalence no longer holds and the cuts are different. The cut strengthening algorithm to be described next can be viewed as an application of the second procedure using the monoid $\mathbb{Z}^{2}$. It can also be viewed as a concretization of the first procedure, which replaces the general definition of the lifting function with a constant time algorithm to compute it.

Let $J_{1}$ be the index set of the integer-constrained variables $s_{j}$, and let $J_{2}=J \backslash J_{1}$.

\section{Lemma 6.1 If the disjunction}

$$
\left(\begin{array}{l}
-r^{1} s \geq f_{1} \\
-r^{2} s \geq f_{2}
\end{array}\right) \vee\left(\begin{array}{l}
r^{1} s \geq 1-f_{1} \\
-r^{2} s \geq f_{2}
\end{array}\right) \vee\left(\begin{array}{l}
r^{1} s \geq 1-f_{1} \\
r^{2} s \geq 1-f_{2}
\end{array}\right) \vee\left(\begin{array}{l}
-r^{1} s \geq f_{1} \\
r^{2} s \geq 1-f_{2}
\end{array}\right)
$$

where $s \geq 0$ and $s_{j} \in \mathbb{Z}, j \in J_{1} \subseteq J$, is valid for $P_{I}$, then so is the disjunction obtained from (6.1) by replacing some or all $r_{j}^{i}, i=1,2, j \in J_{1}$, with $r_{j}^{i}-m_{j}^{i}$, for any $m_{j}^{i} \in \mathbb{Z}, i=1,2, j \in J_{1}$.

Proof Suppose there exists $i_{*} \in\{1,2\}$ and $j_{*} \in J_{1}$ such that replacing $r_{j_{*}}^{i_{*}}$ with $r_{j_{*}}^{i_{*}}-\bar{m}_{j_{*}}^{i_{*}}$, where $\bar{m}_{j_{*}}^{i_{*}} \in \mathbb{Z}$, violates (6.1). Then there exists a solution $(x, s) \in P_{I}$ with $x \in \mathbb{Z}^{2}$ such that

$$
\left(-\left(r_{j_{*}}^{i_{*}}-\bar{m}_{j_{*}}^{i_{*}}\right) s_{j_{*}}-\sum_{j \in J \backslash\left\{j_{*}\right\}} r_{j}^{i_{*}} s_{j}<f_{i_{*}}\right) \wedge\left(\left(r_{j_{*}}^{i_{*}}-\bar{m}_{j_{*}}^{i_{*}}\right) s_{j_{*}}+\sum_{j \in J \backslash\left\{j_{*}\right\}} r_{j}^{i_{*}} s_{j}<1-f_{i_{*}}\right)
$$

holds. Rewriting this expression so as to bring together the terms in $\bar{m}_{j_{*}}^{i_{*}}$ we get

$$
\sum_{j \in J} r_{j}^{i_{*}} s_{j}+f_{i_{*}}-1<\bar{m}_{j_{*}}^{i_{*}} s_{j_{*}}<\sum_{j \in J} r_{j}^{i_{*}} s_{j}+f_{i_{*}}
$$

or

$$
-1<\bar{m}_{j_{*}}^{i_{*}}<0
$$

contrary to the fact that both $\bar{m}_{j_{*}}^{i_{*}}$ and $s_{j_{*}}$ are integer. 
The operation of replacing $r_{j}^{i}$ by $r_{j}^{i}-m_{j}^{i}$ for some $m_{j}^{i} \in \mathbb{Z}, i=1,2$, is called the modularization of $r_{j}^{i}$, or more generally, the modularization of the cut $\alpha x \geq 1$.

Theorem 6.2 Given $(\bar{v}, \bar{w}) \geq 0$ defining a parametric octahedron, the cut $\alpha s \geq 1$ can be strengthened to $\bar{\alpha} s \geq 1$ with coefficients $\bar{\alpha}_{j}, j \in J_{1}$ given by the 3 -variable mixed integer program

$$
\begin{aligned}
\min & \alpha_{j} \\
& \alpha_{j}-\bar{v}_{1} m_{j}^{1}-\bar{w}_{1} m_{j}^{2} \geq-r_{j}^{1} \bar{v}_{1}-r_{j}^{2} \bar{w}_{1} \\
& \alpha_{j}+\bar{v}_{2} m_{j}^{1}-\bar{w}_{2} m_{j}^{2} \geq+r_{j}^{1} \bar{v}_{2}-r_{j}^{2} \bar{w}_{2} \\
& \alpha_{j}+\bar{v}_{3} m_{j}^{1}+\bar{w}_{3} m_{j}^{2} \geq+r_{j}^{1} \bar{v}_{3}+r_{j}^{2} \bar{w}_{3} \\
& \alpha_{j}-\bar{v}_{4} m_{j}^{1}+\bar{w}_{4} m_{j}^{2} \geq-r_{j}^{1} \bar{v}_{4}+r_{j}^{2} \bar{w}_{4} \\
& m_{j}^{1}, m_{j}^{2} \in \mathbb{Z} .
\end{aligned}
$$

The coefficients for $j \in J_{2}$ remain unchanged at $\bar{\alpha}_{j}=\alpha_{j}$ as in Proposition 2.2.

Proof Validity of $\bar{\alpha} s \geq 1$ follows from Lemma 6.1.

Theorem 6.3 The mixed integer program (6.2) has an optimal solution $\left(\bar{\alpha}_{j}, \bar{m}_{j}^{1}, \bar{m}_{j}^{2}\right)$ satisfying $\bar{m}_{j}^{i} \in\left\{\left\lfloor\bar{r}_{j}^{i}\right\rfloor,\left\lceil\bar{r}_{j}^{i}\right\rceil\right\}, i=1,2$.

Proof Consider the linear programming relaxation of (6.2), which asks for minimizing the maximum of four linear functions. This is a piece-wise linear convex programming problem whose minimum is attained for $m_{j}^{i}=r_{j}^{i}, i=1,2$, yielding $\alpha_{j}=\alpha_{j}^{1}=$ $\ldots \ldots=\alpha_{j}^{4}=0$. It is not hard to see that the integer optimum occurs at one of the points $\left(m_{j}^{1}, m_{j}^{2}\right) \in\left\{\left(\left\lfloor r_{j}^{1}\right\rfloor,\left\lfloor r_{j}^{2}\right\rfloor\right),\left(\left\lfloor r_{j}^{1}\right\rfloor,\left\lceil r_{j}^{2}\right\rceil\right),\left(\left\lceil r_{j}^{1}\right\rceil,\left\lfloor r_{j}^{2}\right\rfloor\right),\left(\left\lceil r_{j}^{1}\right\rceil,\left\lceil r_{j}^{2}\right\rceil\right)\right\}$. Let $\alpha_{j}\left(m_{j}^{1}, m_{j}^{2}\right)$ denote the objective function value of (6.2).

Branching, say, on $m_{j}^{1}$ creates two subproblems, $S_{1}, S_{2}$, one for $m_{j}^{1}=\left\lfloor r_{j}^{1}\right\rfloor$, the other for $m_{j}^{1}=\left\lceil r_{j}^{1}\right\rceil$, with objective function values $\alpha_{j}\left(\left\lfloor r_{j}^{1}\right\rfloor, r_{j}^{2}\right)$ and $\alpha_{j}\left(\left\lceil r_{j}^{1}\right\rceil, r_{j}^{2}\right)$ for $S_{1}$ and $S_{2}$, respectively, each of which is a lower bound on the value of an integer solution on its branch. Branching on $m_{j}^{2}$ in subproblem $S_{1}$ creates two new subproblems, $S_{3}$ with $\left(m_{j}^{1}, m_{j}^{2}\right)=\left(\left\lfloor r_{j}^{1}\right\rfloor,\left\lfloor r_{j}^{2}\right\rfloor\right)$ and $S_{4}$ with $\left(m_{j}^{1}, m_{j}^{2}\right)=\left(\left\lfloor r_{j}^{1}\right\rfloor,\left\lceil r_{j}^{2}\right\rceil\right)$. Both of these solutions are integer, so both objective function values $\left.\alpha_{j}\left(\left\lfloor r_{j}^{i}\right\rfloor\right),\left\lfloor r_{j}^{2}\right\rfloor\right)$ and $\alpha_{j}\left(\left\lfloor r_{j}^{i}\right\rfloor,\left\lceil r_{j}^{2}\right\rceil\right)$ provide upper bounds on the integer optimum, which means that the subproblem with the larger upper bound can be discarded. Finally, branching on $m_{j}^{2}$ in subproblem $S_{2}$ creates subproblems $S_{5}$ with $\left(m_{j}^{1}, m_{j}^{2}\right)=\left(\left\lceil r_{j}^{1}\right\rceil,\left\lfloor r_{j}^{2}\right\rfloor\right)$ and $S_{6}$ with $\left(m_{j}^{1}, m_{j}^{2}\right)=\left(\left\lceil r_{j}^{1}\right\rceil,\left\lceil r_{j}^{2}\right\rceil\right)$, both of which have integer solutions whose values $\alpha_{j}\left(\left\lceil r_{j}^{1}\right\rceil,\left\lfloor r_{j}^{2}\right\rfloor\right)$ and $\alpha_{j}\left(\left\lceil r_{j}^{1}\right\rceil,\left\lceil r_{j}^{2}\right\rceil\right)$ provide upper bounds on the integer optimum. It follows that the smallest of the objective function values of the three remaining subproblems $S_{5}, S_{6}$ and either $S_{3}$ or $S_{4}$, is the integer optimum. The situation is perfectly analogous if the first branching occurs on $m_{j}^{2}$ instead of $m_{j}^{1}$.

A modularization that replaces $r_{j}^{i}$ with $r_{j}^{i}-m_{j}^{i}$ for some $m_{j}^{i} \in\left\{\left\lfloor r_{j}^{i}\right\rfloor,\left\lceil r_{j}^{i}\right\rceil\right\}$ will be called a standard modularization. Thus Theorem (6.3) says that the mixed integer program (6.2) attains its optimum for a standard modularization; which means that 
the strengthened coefficients $\bar{\alpha}_{j}$ of cut $\bar{\alpha} s \geq 1$ can be computed by a closed form expression involving at most 16 comparisons:

$$
\bar{\alpha}_{j}=\min _{\left(m_{j}^{1}, m_{j}^{2}\right) \in S} \max _{i \in\{1, \ldots, 4\}}\left\{\alpha_{j}^{i}\left(m_{j}^{1}, m_{j}^{2}\right)\right\},
$$

where

$$
S:=\left\{\left(\left\lfloor r_{j}^{1}\right\rfloor,\left\lfloor r_{j}^{2}\right\rfloor\right),\left(\left\lfloor r_{j}^{1}\right\rfloor,\left\lceil r_{j}^{2}\right\rceil\right),\left(\left\lceil r_{j}^{1}\right\rceil,\left\lfloor r_{j}^{2}\right\rfloor\right),\left(\left\lceil r_{j}^{1}\right\rceil,\left\lceil r_{j}^{2}\right\rceil\right)\right\}
$$

and

$$
\alpha_{j}^{i}\left(m_{j}^{1}, m_{j}^{2}\right)= \pm\left(r_{j}^{1}-m_{j}^{1}\right) \bar{v}_{i} \pm\left(r_{j}^{2}-m_{j}^{2}\right) \bar{w}_{i}
$$

with $( \pm, \pm)$ to be interpreted as $(-,-),(+,-),(+,+),(-,+)$ for $i=1,2,3,4$, respectively.

There are some other properties of the strengthened cut $\bar{\alpha} x \geq 1$ that are worth mentioning.

Lemma 6.4 There exists a standard modularization $\bar{r}$ of the ray $r$ such that

$$
0 \leq f_{i}+\bar{r}^{i} \leq 1, \quad i \in\{1,2\}
$$

i.e. the point $(f+\bar{r})$ belongs to $K$.

Proof If $f_{i}+r^{i}-\left\lfloor r^{i}\right\rfloor \leq 1$ then let $m^{i}=\left\lfloor r^{i}\right\rfloor$. Note that the condition $f_{i}+r^{i}-\left\lfloor r^{i}\right\rfloor \geq 0$ follows since $0 \leq f_{i} \leq 1$ and $r^{i}-\left\lfloor r^{i}\right\rfloor \geq 0$. Otherwise $\left(f_{i}+r^{i}-\left\lfloor r^{i}\right\rfloor>1\right)$ let $m^{i}=\left\lceil r^{i}\right\rceil$ and from $f_{i} \leq 1$ and $r^{i}-\left\lfloor r^{i}\right\rfloor \leq 1$ we get $0 \leq f_{i}+r^{i}-\left\lfloor r^{i}\right\rfloor-1=$ $f_{i}+r^{i}-\left\lceil r^{i}\right\rceil \leq 1$.

For $k=1, \ldots, 4$, let $\bar{\alpha}_{j}^{k}$ be obtained from $\alpha_{j}^{k}$ of (5.4) by substituting $\bar{r}_{j}^{i}$ for $r_{j}^{i}, i=$ 1,2 . One can show that each $\bar{\alpha}_{j}^{k}$ is the convex combination of one of the expressions $\frac{-\bar{r}_{j}^{1}}{f_{1}}$ or $\frac{\bar{r}_{j}^{1}}{1-f_{1}}$ with one of the expressions $\frac{-\bar{r}_{j}^{2}}{f_{2}}$ or $\frac{\bar{r}_{j}^{2}}{1-f_{2}}$. To be specific, we have

\section{Lemma 6.5}

$$
\begin{aligned}
\bar{\alpha}_{j}^{1} & =\lambda_{1} \frac{-\bar{r}_{j}^{1}}{f_{1}}+\left(1-\lambda_{1}\right) \frac{-\bar{r}_{j}^{2}}{f_{2}}, \quad \text { with } \lambda_{1}=\bar{v}_{1} f_{1} \\
\bar{\alpha}_{j}^{2} & =\lambda_{2} \frac{\bar{r}_{j}^{1}}{1-f_{1}}+\left(1-\lambda_{2}\right) \frac{-\bar{r}_{j}^{2}}{f_{2}}, \quad \text { with } \lambda_{2}=\bar{v}_{2}\left(1-f_{1}\right) \\
\bar{\alpha}_{j}^{3} & =\lambda_{3} \frac{\bar{r}_{j}^{1}}{1-f_{1}}+\left(1-\lambda_{3}\right) \frac{\bar{r}_{j}^{2}}{1-f_{2}}, \\
\bar{\alpha}_{j}^{4} & =\lambda_{4} \frac{-\bar{r}_{j}^{1}}{f_{1}}+\left(1-\lambda_{4}\right) \frac{\bar{r}_{j}^{2}}{1-f_{2}}, \quad \text { with } \lambda_{3}=\bar{v}_{3}\left(1-f_{1}\right)
\end{aligned}
$$

Proof By substituting for the $\lambda_{k}, k=1, \ldots, 4$, we get the corresponding expressions for $\bar{\alpha}_{j}^{k}$.

Theorem 6.6 The strengthened cut $\bar{\alpha} s \geq 1$ satisfies $0 \leq \bar{\alpha}_{j} \leq 1, j \in J_{1}$. 
Proof Since $\bar{v}_{k}, \bar{w}_{k} \geq 0$ for all $k$, we have $\bar{\alpha}_{j}^{k} \geq 0$ for at least one of the four $k$, hence $\bar{\alpha}_{j} \geq 0$. Let $\left(\bar{\alpha}, \bar{m}^{1}, \bar{m}^{2}\right)$ be an optimal solution to (6.2). Let $\bar{r}^{i}=r^{i}-\bar{m}^{i}, i=1,2$, where $\bar{m}^{i} \in\left\{\left\lfloor r^{i}\right\rfloor,\left\lceil r^{i}\right\rceil\right\} i=1,2$. There are four cases:

Case 1. $\bar{m}^{i}=\left\lfloor r^{i}\right\rfloor, i=1,2$. Then $\bar{r}^{i}=r^{i}-\bar{m}^{i} \geq 0, i=1,2$, and $\bar{\alpha}^{1}=-\bar{r}^{1} \bar{v}_{1}-\bar{r}^{2} \bar{w}_{1} \leq 0$.

$\bar{\alpha}^{2}=\bar{r}^{1} \bar{v}_{2}-\bar{r}^{2} \bar{w}_{2} \leq \bar{r}^{1} /\left(1-f_{1}\right)\left(\right.$ from (5.3)). From Lemma $6.4, \bar{r}^{1} /\left(1-f_{1}\right) \leq 1$, hence $\bar{\alpha}^{2} \leq 1$.

$\bar{\alpha}^{3}=\bar{r}^{1} \bar{v}_{3}+\bar{r}^{2} \bar{w}_{3}=\lambda_{3} \bar{r}^{1} /\left(1-f_{1}\right)+\left(1-\lambda_{3}\right) \bar{r}^{2} /\left(1-f_{2}\right)$, with $\lambda_{3}=\bar{v}_{3}\left(1-f_{1}\right)$ (from Lemma 6.5). But from Lemma $6.4, \bar{r}^{i} /\left(1-f_{i}\right) \leq 1, i=1,2$, hence $\bar{\alpha}^{3} \leq 1$.

$\bar{\alpha}^{4}=-\bar{r}^{1} \bar{v}_{4}+\bar{r}^{2} \bar{w}_{4} \leq \bar{r}^{2} /\left(1-f_{2}\right) \leq 1$ (from (6.3), hence $\bar{\alpha}^{4} \leq 1$.

The remaining three cases, namely $\left(\bar{m}^{1}, \bar{m}^{2}\right)=\left(\left\lceil\bar{r}^{1}\right\rceil,\left\lfloor\bar{r}^{2}\right\rfloor\right),\left(\bar{m}^{1}, \bar{m}^{2}\right)=\left(\left\lfloor\bar{r}^{1}\right\rfloor,\left\lceil\bar{r}^{2}\right\rceil\right)$, and $\bar{m}^{i}=\left(\left\lceil\bar{r}^{1}\right\rceil,\left\lceil\bar{r}^{2}\right\rceil\right), i=1,2$, are similar.

In the following we illustrate the cut strengthening procedure on a small numerical instance.

Example Consider the instance

$$
\begin{aligned}
x_{1} & =\frac{1}{3}+\frac{4}{3} s_{1}+\frac{13}{2} s_{2}-\frac{9}{4} s_{3}-\frac{4}{3} s_{4} \\
x_{2} & =\frac{1}{3}+\frac{7}{2} s_{1}-\frac{7}{3} s_{2}-\frac{7}{6} s_{3}+\frac{5}{4} s_{4} \\
s_{j} & \geq 0 j j \in J \\
x_{1}, x_{2}, s_{j} & \in \mathbb{Z} j \in J
\end{aligned}
$$

\begin{tabular}{|c|c|c|c|c|c|c|}
\hline $15 \mathrm{~s}$ & & $7 s_{2}$ & + & $3.5 s_{3}$ & + & $2.4667 s_{4}$ \\
\hline & & $7 s_{2}$ & + & $3.5 s_{3}$ & + & $1.875 s_{4}$ \\
\hline ut $3: 2 s_{1}$ & + & $9.75 s_{2}$ & + & $3.5 s_{3}$ & + & $2.6216 s_{4}$ \\
\hline
\end{tabular}

where $J=\{1 \ldots 4\}$.

We first relax the integrality constraints $s_{j} \in \mathbb{Z}, j \in J$ and derive the three facet defining inequalities of $P_{D}$ :

These intersection cuts come from a triangle of type B, a horizontal strip, and a triangle of type A, respectively, with associated $v, w$ values shown below:

$$
\begin{aligned}
& \text { Cut } 1: v=\left[\begin{array}{llll}
0 & 0 & 1.1887 & 0.8353
\end{array}\right] ; \quad w=\left[\begin{array}{llll}
3 & 3 & 0.3113 & 1.0824
\end{array}\right] \\
& \text { Cut } 2: v=\left[\begin{array}{lllll}
0 & 0 & 0 & 0 & ] ;
\end{array} \quad w=\left[\begin{array}{llll}
3 & 3 & 1.5 & 1.5
\end{array}\right]\right. \\
& \text { Cut } 3: v=\left[\begin{array}{llll}
0 & 1.5 & 1.5 & 1.0541
\end{array}\right] ; \quad w=\left[\begin{array}{llll}
3 & 0 & 0 & 0.9730
\end{array}\right] \text {. }
\end{aligned}
$$

We now apply the strengthening procedure to Cut 1 .

- For the ray $\left(r_{1}^{1}, r_{1}^{2}\right)=\left(\frac{4}{3}, \frac{7}{2}\right)$ :

- choosing $\left(m_{1}^{1}, m_{1}^{2}\right)=\left(\left\lfloor r_{1}^{1}\right\rfloor,\left\lfloor r_{1}^{2}\right\rfloor\right)=(1,3)$ yields $\bar{\alpha}_{1}=0.5519$

- choosing $\left(m_{1}^{1}, m_{1}^{2}\right)=\left(\left\lfloor r_{1}^{1}\right\rfloor,\left\lceil r_{1}^{2}\right\rceil\right)=(1,4)$ yields $\bar{\alpha}_{1}=1.5$ 
- choosing $\left(m_{1}^{1}, m_{1}^{2}\right)=\left(\left\lceil r_{1}^{1}\right\rceil,\left\lfloor r_{1}^{2}\right\rfloor\right)=(2,3)$ yields $\bar{\alpha}_{1}=1.0981$

- choosing $\left(m_{1}^{1}, m_{1}^{2}\right)=\left(\left\lceil r_{1}^{1}\right\rceil,\left\lceil r_{1}^{2}\right\rceil\right)=(2,4)$ yields $\bar{\alpha}_{1}=1.5$.

The minimum is attained for $\left(m_{1}^{1}, m_{1}^{2}\right)=\left(\left\lfloor r_{1}^{1}\right\rfloor,\left\lfloor r_{1}^{2}\right\rfloor\right)=(1,3)$ that produces $\alpha_{1}=$ 0.5519 .

- For the ray $\left(r_{2}^{1}, r_{2}^{2}\right)=\left(\frac{13}{2},-\frac{7}{3}\right)$, the minimum is attained for $\left(m_{2}^{1}, m_{2}^{2}\right)=$ $\left(\left\lfloor r_{2}^{1}\right\rfloor,\left\lfloor r_{2}^{2}\right\rfloor\right)=(6,-3)$ that produces $\bar{\alpha}_{2}=0.8019$.

- For the ray $\left(r_{3}^{1}, r_{3}^{2}\right)=\left(-\frac{9}{4},-\frac{7}{6}\right)$, the minimum is attained for $\left(m_{3}^{1}, m_{3}^{2}\right)=$ $\left(\left\lceil r_{3}^{1}\right\rceil,\left\lceil r_{3}^{2}\right\rceil\right)=(-2,-1)$ that produces $\bar{\alpha}_{3}=0.5$.

- For the ray $\left(r_{3}^{1}, r_{4}^{2}\right)=\left(-\frac{4}{3}, \frac{5}{4}\right)$, the minimum is attained for $\left(m_{4}^{1}, m_{4}^{2}\right)=$ $\left(\left\lceil r_{4}^{1}\right\rceil,\left\lfloor r_{4}^{2}\right\rfloor\right)=(-1,1)$ that produces $\bar{\alpha}_{4}=0.549$.

So we obtained the strengthened cut

$$
0.5519 s_{1}+0.8019 s_{2}+0.5 s_{3}+0.549 s_{4} \geq 1 .
$$

After applying the same procedure to the cuts 2 and 3 we get the following strengthened cuts:

$$
\begin{aligned}
& \text { Strengthened cut } 1: 0.5519 s_{1}+0.8019 s_{2}+0.5 s_{3}+0.549 s_{4} \geq 1 \\
& \text { Strengthened cut } 2: 0.75 s_{1}+1 s_{2}+0.5 s_{3}+0.375 s_{4} \geq 1 \\
& \text { Strengthened cut } 3: 0.5 s_{1}+0.75 s_{2}+0.5 s_{3}+0.5946 s_{4} \geq 1 .
\end{aligned}
$$

We can compare these cuts with the Mixed Integer Gomory (GMI) cuts that are obtained from $x_{1}, x_{2}$ :

$$
\begin{aligned}
& \text { GMI from } x_{1}: 0.5 s_{1}+0.75 s_{2}+0.75 s_{3}+1 s_{4} \geq 1 \\
& \text { GMI from } x_{2}: 0.75 s_{1}+1 s_{2}+0.5 s_{3}+0.375 s_{4} \geq 1
\end{aligned}
$$

Notice that the GMI cut derived from $x_{1}$ is dominated by the strengthened cut 3 and the GMI cut derived from $x_{2}$ is the same as the strengthened cut 2.

Another way to use the integrality of the $s_{j}, j \in J_{1}$, to obtain different and often stronger cuts consists in the following three-step procedure:

1. Apply standard modularization to the two rows from which the cut is generated [e.g., as in Lemma 6.4, replace each ray $r^{j}, j \in J_{1}$, with $\bar{r}^{j}$ satisfying (6.3)].

2. Generate a cut $\alpha x \geq 1$ from the two modularized rows.

3. Modularize the resulting cut with the choice of $\bar{m}_{j}^{1}, \bar{m}_{j}^{2}$ given by Theorems 6.2-6.3, to obtain the strengthened cut $\alpha x \geq 1$.

Since step 1 replaces the original rays $r^{j}, j \in J_{1}$ with the new, modularized rays $\bar{r}^{j}$, the basic solutions of the resulting CGLP, and hence the parameters $(v, w)$, are different from the original ones. Therefore the cuts obtained in step 2 are new. The modularization of step 3 may or may not strengthen them, but they are different from the ones obtained from the original unmodularized $r^{j}$. 
Yet another way to use the integrality of the variables $s_{j}, j \in J_{1}$, is to apply the monoidal cut strengthening procedure of Balas and Jeroslow (1980) with a monoid different from $\mathbb{Z}^{2}$. For cuts generated from a disjunction of the form (6.1), this procedure involves the use of lower bounds on the expressions on the left-hand side of each inequality. While these bounds are readily available and quite tight in the case when $x_{1}, x_{2} \in\{0,1\}$, they can be weak in the general case of $x_{1}, x_{2} \in \mathbb{Z}$. We therefore defer the discussion of this procedure until the (next) section on the $0-1$ disjunctive hull.

\section{The 0-1 disjunctive hull}

We now consider the 0-1 disjunctive hull $P_{D}^{=}$for $q=2$, i.e. we work with $P_{01}=$ $\left\{(x, s) \in\{0,1\}^{2} \times \mathbb{R}^{|J|}:(x, s) \in P_{L}\right\}$ where $P_{L}$ is given in (5.1). The CGLP that produces the facets of $P_{D}^{=}$is the linear program with the constraint set of Theorem 2.5. In addition to the four configurations of the parametric octahedron for the MIP CGLP given in Sec. 5, when $v, w$ are unrestricted in sign some additional configurations are possible: (a) triangles with each face containing exactly one vertex of $K$, which we call triangles of type $\mathrm{C}\left(T_{C}\right)$; and (b) cones, designated as $(C)$.

Note that our triangles of type $\mathrm{C}$ are similar to the class of triangles of type 3 for cuts for mixed integer programs described in Dey and Wolsey (2010). The difference between these classes is that on the one hand, the three integer points contained in the faces of triangles of type 3 defined in Dey and Wolsey (2010) need not be vertices of $K$; on the other hand, our triangles of type $\mathrm{C}$ may also contain (non-0-1) integer points, positive or negative, in their interior. The presence among the parametric octahedra of unbounded ones, namely cones, implies that the cuts $\alpha s \geq 1$ of this class may have coefficients $\alpha_{j}<0$.

As we did in Sec. 5, we give a classification of the parametric cross-polytopes that correspond to disjunctive hull facets for the $0-1$ case (i.e. facets of $P_{D}^{=}$). Let $k_{1} \in$ $\{1, \ldots, 4\}$ be the index of any vertex of $K$. We denote by $k_{2}, k_{3}, k_{4}$ the indices of the vertices of $K$ that follow $k_{1}$ in counter-clockwise order. The following configurations, in addition to those for facets of $P_{D}$, are exhaustive when considering every value for $k_{1} \in\{1, \ldots, 4\}(\bmod 4)$ and swapping $v_{i}$ with $w_{i}$. In each case, the shape of $P_{\text {octa }}$ is determined by a strict subset of the four pairs $\left(v_{i}, w_{i}\right)$, the remaining pairs being inactive.

- $\left(T_{C 1}\right) v_{k_{1}}>0, w_{k_{1}}<0 ; v_{k_{2}}, w_{k_{2}}>0 ; v_{k_{3}}>0, w_{k_{3}}>0,\left(v_{k_{4}}, w_{k_{4}}>0\right) . P_{\text {octa }}$ is a triangle of type $C$ with all its vertices outside the cube $K$. The face corresponding to $k_{4}$ is inactive. See Fig. $4 \mathrm{a}$.

- $\left(T_{C 2}\right) v_{k_{1}}>0, w_{k_{1}}<0 ; v_{k_{2}}, w_{k_{2}}>0 ; v_{k_{3}}<0, w_{k_{3}}>0,\left(v_{k_{4}}, w_{k_{4}}>0\right)$. $P_{\text {octa }}$ is a triangle of type $\mathrm{C}$ with one vertex in the cube $K$. The face corresponding to $k_{4}$ is inactive. See Fig. $4 \mathrm{~b}$.

- $\left(C_{A}\right) v_{k_{2}}, v_{k_{3}}>0 ; w_{k_{2}}=w_{k_{3}}=0, v_{k_{4}}>0, w_{k_{4}}<0,\left(v_{k_{1}}, w_{k_{1}}>0\right)$. $P_{\text {octa }}$ is a cone with one face containing two adjacent vertices of $K$, the other face containing one vertex of $K$. The face corresponding to $k_{1}$ is inactive. See Fig. $4 \mathrm{c}$.

- $\left(C_{B}\right) v_{k_{1}}<0, w_{k_{1}}>0 ; v_{k_{3}}>0, w_{k_{3}}<0, v_{k_{4}}, w_{k_{4}}>0,\left(v_{k_{2}}, w_{k_{2}}>0\right)$. $P_{\text {octa }}$ is a cone with one face containing two nonadjacent vertices of $K$, the other face containing one vertex of $K$. The face corresponding to $k_{2}$ is inactive. See Fig. $4 d$. 


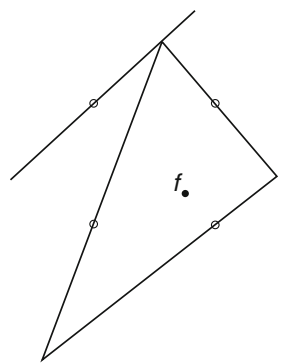

(a) triangle of type $\mathrm{C}$ with all vertices outside $K$

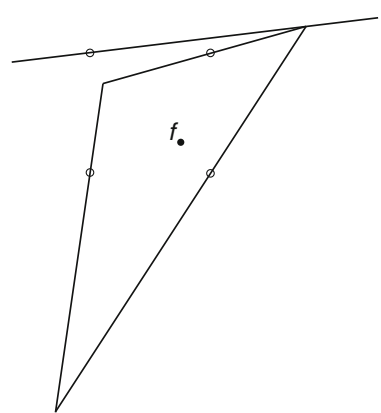

(b) triangle of type $\mathrm{C}$ with one vertex inside $K$

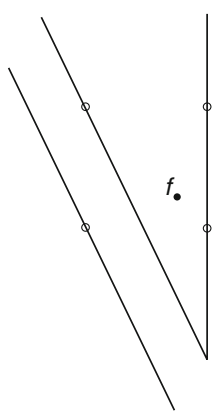

(c) cone with one face containing two adjacent vertices of $K$

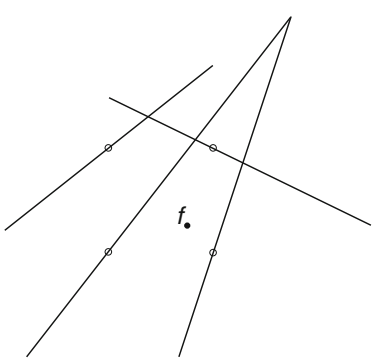

(f) truncated cone with each face containing one vertex of $K$ (d) cone with one face containing two nonadjacent vertices of $K$

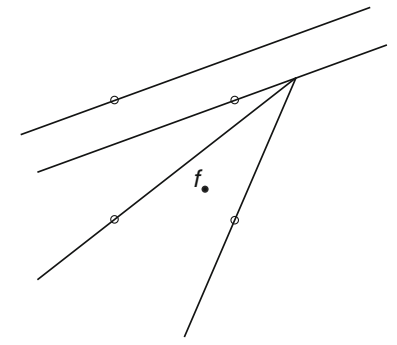

(e) cone with each face containing one vertex of $K$

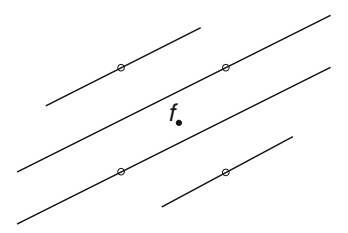

(g) tilted strip

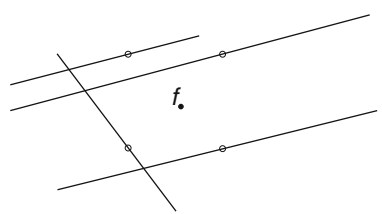

(h) truncated tilted strip

Fig. 4 Additional configurations of the parametric octahedron for the 0-1 case

- $\left(C_{C}\right) v_{k_{1}}>0, w_{k_{1}}<0 ; v_{k_{2}}, w_{k_{2}}>0 ;\left(v_{k_{3}}<0, w_{k_{3}}>0\right),\left(v_{k_{4}}, w_{k_{4}}>0\right)$. $P_{\text {octa }}$ is a cone with each face containing one vertex of $K$. The faces corresponding to $k_{3}$ and $k_{4}$ are inactive. See Fig. 4 e.

- $\left(C_{C T}\right) v_{k_{1}}>0, w_{k_{1}}<0 ; v_{k_{2}}, w_{k_{2}}>0 ; v_{k_{3}}, w_{k_{3}}>0 ;\left(v_{k_{4}}, w_{k_{4}}>0\right)$. $P_{\text {octa }}$ is a truncated cone with each face containing one vertex of $K$. The face corresponding to $k_{4}$ is inactive. See Fig. $4 \mathrm{f}$.

- (S) $v_{k_{1}}<0, w_{k_{1}}>0 ; v_{k_{3}}>0, w_{k_{3}}<0,\left(v_{k_{2}}, w_{k_{2}}>0, v_{k_{4}}, w_{k_{4}}>0\right)$. $P_{\text {octa }}$ is a tilted strip, each side of which contains one vertex of $K$. The faces corresponding to the remaining two vertices are inactive. See Fig. $4 \mathrm{~g}$. 
- $\left(S_{T}\right) v_{k_{1}}, w_{k_{1}}>0 ; v_{k_{2}}, w_{k_{2}}>0 ; v_{k_{3}}>0, w_{k_{3}}<0 ;\left(v_{k_{4}}, w_{k_{4}}>0\right)$. $P_{\text {octa }}$ is a truncated (tilted) strip, each side of which contains a vertex of $K$ The face corresponding to $k_{4}$ is inactive. See Fig. $4 \mathrm{~h}$.

Example Consider the Andersen et al. (2007) instance, amended with the condition $x_{i} \in\{0,1\}, i \in\{1,2\}$ :

$$
\begin{array}{lllll}
x_{1}=\frac{1}{4}+2 s_{1}+1 s_{2} & -3 s_{3} & +1 s_{5} \\
x_{2}=\frac{1}{2}+1 s_{1}+1 s_{2}+2 s_{3} & -1 s_{4} & -2 s_{5} \\
x_{1}, x_{2} \in\{0,1\}, s \geq 0 . & &
\end{array}
$$

In Sec. 5 we listed the five cuts defining the facets of the disjunctive hull for this example, without the 0-1 condition. Using the stronger disjunction expressing the $0-1$ condition we obtain the following 12 cuts that define the facets of conv $P_{D}^{=}$.

1. Cut (type $S$ ): $2.667 s_{1}+1.333 s_{2}+12 s_{3}+0 s_{4}+1.333 s_{5} \geq 1$

$$
\begin{aligned}
& v_{1}=4 ; \quad v_{2}=1.333 ; v_{3}=1.333 ; v_{4}=4 \\
& w_{1}=0 ; \quad w_{2}=0 ; w_{3}=0 ; w_{4}=0
\end{aligned}
$$

2. Cut (type $T_{B}$ ): $2.667 s_{1}+1.333 s_{2}+4.889 s_{3}+0.8889 s_{4}+1.333 s_{5} \geq 1$

$$
\begin{aligned}
& v_{1}=2.222 ; v_{2}=1.333 ; v_{3}=1.333 ; v_{4}=0.4444 \\
& w_{1}=0.8889 ; \quad w_{2}=0 ; \quad w_{3}=0 ; \quad w_{4}=1.778
\end{aligned}
$$

3. Cut (type $T_{B}$ ): $2 s_{1}+2 s_{2}+4 s_{3}+1 s_{4}+1.714 s_{5} \geq 1$

$v_{1}=2 ; v_{2}=1.143 ; v_{3}=0 ; v_{4}=0$

$w_{1}=1 ; w_{2}=0.2857 ; w_{3}=2 ; w_{4}=2$

4. Cut (type $\left.T_{C 1}\right): 2.947 s_{1}+1.053 s_{2}+5.263 s_{3}+0.8421 s_{4}+3.579 s_{5} \geq 1$

$v_{1}=2.316 ; v_{2}=0.7719 ; v_{3}=1.895 ; v_{4}=0.6316$

$w_{1}=0.8421 ; w_{2}=0.8421 ; w_{3}=-0.8421 ; w_{4}=1.684$

5. Cut (type $T_{C 1}$ ): $1.63 s_{1}+2.37 s_{2}+8.444 s_{3}+0.4444 s_{4}+1.926 s_{5} \geq 1$

$v_{1}=3.111 ; v_{2}=1.037 ; v_{3}=-0.7407 ; v_{4}=2.222$

$w_{1}=0.4444 ; w_{2}=0.4444 ; w_{3}=3.111 ; w_{4}=0.8889$

6. Cut (type $T_{C 2}$ ): $4.364 s_{1}+2.545 s_{2}+3.273 s_{3}+1.091 s_{4}+0.3636 s_{5} \geq 1$

$$
\begin{aligned}
& v_{1}=1.818 ; v_{2}=1.818 ; v_{3}=1.818 ; v_{4}=-0.3636 \\
& w_{1}=1.091 ; \quad w_{2}=-0.7273 ; w_{3}=0.7273 ; w_{4}=2.182
\end{aligned}
$$


7. Cut (type $T_{C 2}$ ): $3.765 s_{1}+3.059 s_{2}+2.588 s_{3}+1.176 s_{4}+0.7059 s_{5} \geq 1$

$$
\begin{aligned}
& v_{1}=1.647 ; v_{2}=1.647 ; v_{3}=0.7059 ; v_{4}=-0.7059 \\
& w_{1}=1.176 ; w_{2}=-0.4706 ; w_{3}=2.353 ; w_{4}=2.353
\end{aligned}
$$

8. Cut (type $C_{A}$ ): $12 s_{1}+8 s_{2}+12 s_{3}+0 s_{4}-4 s_{5} \geq 1$

$$
\begin{aligned}
& v_{1}=4 ; v_{2}=4 ; v_{3}=4 ; v_{4}=4 \\
& w_{1}=0 ; w_{2}=-4 ; w_{3}=4 ; w_{4}=0
\end{aligned}
$$

9. Cut (type $C_{B}$ ): $32 s_{1}+20 s_{2}-20 s_{3}+4 s_{4}+12 s_{5} \geq 1$

$$
\begin{aligned}
& v_{1}=-4 ; v_{2}=4 ; v_{3}=4 ; v_{4}=-12 \\
& w_{1}=4 ; w_{2}=4 ; \quad w_{3}=-4 ; w_{4}=8
\end{aligned}
$$

10. Cut (type $C_{B}$ ): $12 s_{1}+8 s_{2}+44 s_{3}-4 s_{4}-4 s_{5} \geq 1$

$$
\begin{aligned}
& v_{1}=12 ; v_{2}=4 ; v_{3}=4 ; v_{4}=-4 \\
& w_{1}=-4 ; w_{2}=-4 ; w_{3}=4 ; w_{4}=4
\end{aligned}
$$

11. Cut (type $C_{C}$ ): $-2 s_{1}+6 s_{2}+52 s_{3}+2 s_{4}+4 s_{5} \geq 1$

$$
\begin{aligned}
& v_{1}=0 ; \quad v_{2}=0 ; \quad v_{3}=-8 ; v_{4}=8 \\
& w_{1}=2 ; w_{2}=2 ; \quad w_{3}=14 ; w_{4}=-2
\end{aligned}
$$

12. Cut (type $C_{B}$ ): $8 s_{1}-4 s_{2}+12 s_{3}+16 s_{4}+44 s_{5} \geq 1$

$$
\begin{aligned}
& v_{1}=4 ; v_{2}=-9.333 ; v_{3}=12 ; v_{4}=4 \\
& w_{1}=0 ; w_{2}=16 ; w_{3}=-16 ; w_{4}=0 .
\end{aligned}
$$

The above list of 12 cuts includes 3 of the 5 cuts defining facets of conv $P_{D}$, namely 1, 2 and 4, which appear on our list in position 3,2 and 1, respectively. The remaining two facets of conv $P_{D}$, given by cuts 3 and 5, are redundant for conv $P_{D}^{=}$; namely, cut 3 is a convex combination of cuts 2, 3, 6 and 7 on our list, while cut 5 is a convex combination of cuts 1 and 5 on our list.

The number of facets of conv $P_{\bar{D}}^{\bar{D}}$ substantially exceeds the number of facets of conv $P_{D}$. In order to assess the impact of the two sets of cuts, we computed the average integrality gap on (7.1) for 1,000 randomly generated objective functions. Adding the five cuts valid for the two-row MIP reduces this gap by $77 \%$; while adding the additional cuts valid for the $0-1$ case reduces $100 \%$ of the gap.

Next we discuss the strengthening of valid cuts for $P_{D}^{=}$when some variables $s_{j}$ are integer-constrained. Let $J_{1}$ be the index set of such variables.

First of all, we observe that the standard modularization procedure described in Theorem 6.2 for strengthening cuts for $P_{D}$ is not valid in the case of cuts for $P_{D}^{=}$ (except of course for the case of those cuts for $P_{D}^{=}$that are also valid for $P_{D}$ ). Indeed, Lemma 6.1 which underlies the correctness of the procedure in the case of $P_{D}$ is no longer valid in the case of $P_{D}^{=}$: if the disjunction (6.1) is modified by replacing every 
inequality with equality, then it is no longer equivalent to the disjunction obtained by replacing $r_{j}^{i}$ with $r_{j}^{i}-m_{j}^{i}$ for some $m_{j}^{i} \in \mathbb{Z}$. Instead, we will use monoidal strengthening with a monoid different from $\mathbb{Z}^{2}$.

Consider a disjunction of the form

$$
\bigvee_{k \in Q}\left(A^{k} x \geq a_{0}^{k}\right), \quad A^{k}=\left(a_{j}^{k}\right), j \in J, \quad a_{j}^{k} \in \mathbb{R}^{m}, j \in J \cup\{0\},
$$

and the valid cut $\alpha x \geq 1$, where

$$
\alpha_{j}=\max _{k \in Q}\left\{\theta^{k} a_{j}^{k} / \theta^{k} a_{0}^{k}\right\}
$$

for some $\theta^{k} \in \mathbb{R}_{+}^{m}, k \in Q$.

Suppose now that for each $A^{k} x, k \in Q$, a lower bound $b_{0}^{k} \leq a_{0}^{k}$ is known, i.e. $A^{k} x \geq b_{0}^{k}, k \in Q$.

Theorem 7.1 Let $M:=\left\{m \in \mathbb{Z}^{|Q|}: \sum_{k \in Q} m^{k} \geq 0\right\}$. If $x_{j} \in \mathbb{Z}, j \in J_{1}$, then the cut $\alpha x \geq 1$ can be strengthened to $\bar{\alpha} x \geq 1$, where

$$
\bar{\alpha}_{j}=\min _{m_{j} \in M} \max _{k \in Q}\left\{\left(\theta^{k} a_{j}^{k}+m_{j}^{k} \theta^{k}\left(a_{0}^{k}-b_{0}^{k}\right)\right) / \theta^{k} a_{0}^{k}\right\} \quad j \in J_{1}
$$

and $\bar{\alpha}_{j}=\alpha_{j}$ for $j \in J \backslash J_{1}$.

Proof See Balas and Jeroslow (1980) or Balas (1979).

We will now apply this Theorem to our case, i.e. to strengthen an inequality valid for $P_{D}^{=}$. Such an inequality, say $\alpha s \geq 1$, is derived from the disjunction

$$
\left(\begin{array}{c}
-r^{1} s=f_{1} \\
-r^{2} s=f_{2}^{1}
\end{array}\right) \vee\left(\begin{array}{c}
r^{1} s=1-f \\
-r^{2} s=f_{2}
\end{array}\right) \vee\left(\begin{array}{c}
r^{1} s=1-f_{1} \\
r^{2} s=1-f_{2}
\end{array}\right) \vee\left(\begin{array}{c}
-r^{1} s=f_{1} \\
r^{2} s=1-f_{2}
\end{array}\right) .
$$

Using Theorem 2.5 we see that the cut $\alpha s \geq 1$ from (7.5) differs from the cut derived from the corresponding disjunction which has inequalities instead of equations, only in that the variables $v_{k}, w_{k}$ are unrestricted in sign. In other words, the cut coefficients $\alpha_{j}$ are those given by (5.4), restated here as

$$
\alpha_{j}=\max _{i=\{1, \ldots, 4\}}\left\{\alpha_{j}^{i}\right\}
$$

with

$$
\begin{aligned}
& \alpha_{j}^{1}=-r_{j}^{1} \bar{v}_{1}-r_{j}^{2} \bar{w}_{1}, \\
& \alpha_{j}^{2}=r_{j}^{1} \bar{v}_{2}-r_{j}^{2} \bar{w}_{2}, \\
& \alpha_{j}^{3}=r_{j}^{1} \bar{v}_{3}+r_{j}^{2} \bar{w}_{3}, \\
& \alpha_{j}^{4}=-r_{j}^{1} \bar{v}_{4}+r_{j}^{2} \bar{w}_{4} .
\end{aligned}
$$


where the parameters $\left(\bar{v}_{k}, \bar{w}_{k}\right)$ come from an optimal solution to a (CGLP) with constraint set (5.3) without the condition $\left(v_{i}, w_{i}\right) \geq 0, i \in\{1, \ldots, 4\}$. Clearly, the absence of these nonnegativity constraints implies that $\alpha_{j}$ may also be negative.

In order to apply Theorem 7.1 to this cut $\alpha s \geq 1$, we need to compute lower bounds on the left-hand side of each equation in (7.5). For this wee need to restate (7.5)as a disjunction between systems of inequalities rather than equations, i.e. we need to replace the first term of (7.5) with

$$
\left(\begin{array}{rl}
-r^{1} s & \geq f_{1} \\
r^{1} s & \geq-f_{1} \\
-r^{2} s & \geq f_{2} \\
r^{2} s & \geq-f_{2}
\end{array}\right)
$$

and so on. In this setting, the vectors $a_{0}^{k}$ of Theorem 7.1 are

$$
a_{0}^{1}=\left(\begin{array}{r}
f_{1} \\
-f_{1} \\
f_{2} \\
-f_{2}
\end{array}\right), \quad a_{0}^{2}=\left(\begin{array}{c}
1-f_{1} \\
f_{1}-1 \\
f_{2} \\
-f_{2}
\end{array}\right), \quad a_{0}^{3}=\left(\begin{array}{c}
1-f_{1} \\
f_{1}-1 \\
1-f_{2} \\
f_{2}-1
\end{array}\right), \quad a_{0}^{4}=\left(\begin{array}{c}
f_{1} \\
-f_{2} \\
1-f_{2} \\
f_{2}-1
\end{array}\right)
$$

and the lower bounds $b_{0}^{k}$ are

$$
b_{0}^{1}=\left(\begin{array}{c}
f_{1}-1 \\
-f_{1} \\
f_{2}-1 \\
-f_{2}
\end{array}\right), \quad b_{0}^{2}=\left(\begin{array}{c}
-f_{1} \\
f_{1}-1 \\
f_{2}-1 \\
-f_{2}
\end{array}\right), \quad b_{0}^{3}=\left(\begin{array}{c}
-f_{1} \\
f_{1}-1 \\
-f_{2} \\
f_{2}-1
\end{array}\right), \quad b_{0}^{4}=\left(\begin{array}{c}
f_{1}-1 \\
-f_{1} \\
-f_{2} \\
f_{2}-1
\end{array}\right)
$$

Also, the parameters (multipliers) $\left(v_{k}, w_{k}\right)$, unrestricted in sign, need to be replaced with nonnegative parameters $\left(v_{k}^{\prime}, v_{k}^{\prime \prime}, w_{k}^{\prime}, w_{k}^{\prime \prime}\right), k=1, \ldots, 4$, where $v_{k}=v_{k}^{\prime}-v_{k}^{\prime \prime}$ and $w_{k}=w_{k}^{\prime}-w_{k}^{\prime \prime}$. The vectors $\left(v_{k}^{\prime}, v_{k}^{\prime \prime}, w_{k}^{\prime}, w_{k}^{\prime \prime}\right)$ play the role of $\theta_{k}$ in Theorem 7.1.

We can now apply Theorem 7.1 to our case. Define $\bar{v}_{k}^{+}:=\max \left\{\bar{v}_{k}, 0\right\}, \bar{w}_{k}^{+}=$ $\max \left\{\bar{w}_{k}, 0\right\}, k=1, \ldots, 4$. Then we have

Corollary 7.2 Let $M=\left\{m \in \mathbb{Z}^{4}: \sum_{k=1}^{4} m^{k} \geq 0\right\}$. Then $\hat{\alpha} s \geq 1$ is a valid cut for $P_{D}^{=}$, with $\hat{\alpha}_{j}=\max _{k \in\{1, \ldots, 4\}}\left\{\hat{\alpha}_{j}^{k}\right\}$, and

$$
\hat{\alpha}_{j}^{k}= \begin{cases}\min _{m \in M} \max _{k \in\{1, \ldots, 4\}}\left\{\alpha_{j}^{k}+m_{j}^{k}\left(\bar{v}_{k}^{+}+\bar{w}_{k}^{+}\right)\right\}, & j \in J_{1} \\ \alpha_{j}^{k}, & j \in J \backslash J_{1} .\end{cases}
$$


Proof Applying Theorem 7.1 with $a_{0}^{k}, b_{0}^{k}$ as above and $\theta^{k}=\left(\bar{v}_{k}^{\prime}, \bar{v}_{k}^{\prime \prime}, \bar{w}_{k}^{\prime}, \bar{w}_{k}^{\prime \prime}\right), k=$ $1, \ldots, 4$, we have

$$
\begin{gathered}
\theta^{k} a_{j}^{k}=\left\{\begin{array}{c}
-r_{j}^{1} \bar{v}_{1}-r_{j}^{2} \bar{w}_{1}=\alpha_{j}^{1}, k=1 \\
r_{j}^{1} \bar{v}_{2}-r_{j}^{2} \bar{w}_{2}=\alpha_{j}^{2}, k=2 \\
r_{j}^{1} \bar{v}_{3}+r_{j}^{2} \bar{w}_{3}=\alpha_{j}^{3}, k=3 \\
-r_{j}^{1} \bar{v}_{4}+r_{j}^{2} \bar{w}_{4}=\alpha_{j}^{4}, k=4
\end{array} \theta^{k} a_{0}^{k}\right. \\
\theta^{k}\left(a_{0}^{k}-b_{0}^{k}\right)=\left(\bar{v}_{k}^{\prime}, \bar{v}_{k}^{\prime \prime}, \bar{w}_{k}^{\prime}, \bar{w}_{k}^{\prime \prime}\right)\left(\begin{array}{l}
1 \\
0 \\
1 \\
0
\end{array}\right) \\
=\left(\bar{v}_{k}^{\prime}+\bar{w}_{k}^{\prime}\right)=\left(\bar{v}_{k}^{+}+\bar{w}_{k}^{+}\right) .
\end{gathered}
$$

The expression for $\hat{\alpha}_{j}^{k}$ in the Corollary now follows by substitution.

Finding the optimal $m_{j}^{k} \in M$ requires a small (single digit) number of comparisons. While Balas and Jeroslow (1980) and Balas (1979) give simple procedures for the case of a general disjunction, the optimal $m_{j}^{k}$ of Corollary 7.2 for a given $j \in J_{1}$ can be found as follows:

- Start with $m_{j}^{k}=0, \widetilde{\alpha}_{j}^{k}=\widetilde{\alpha}_{j}^{k}$ for all $k$ and apply the

\section{Iterative step.}

- Find $\alpha_{j}^{\max }=\max _{k} \widetilde{\alpha}_{j}^{k}, \alpha_{j}^{\min }=\min _{k} \widetilde{\alpha}_{j}^{k}$ and let $m_{j}^{\max }, m_{j}^{\min }$ be the corresponding values of $m_{j}^{k}$.

- Set $m_{j}^{\max }=m_{j}^{\max }-t, m_{j}^{\min }=m_{j}^{\min }+t$, where $t$ is the smallest positive integer for which the identity of $\widetilde{\alpha}_{j}^{\max }$ changes.

- If the value of $\max _{k} \widetilde{\alpha}_{j}^{k}$ has not been reduced, stop with $m_{j}^{\max }=m_{j}^{\max }-t+1$, $m_{j}^{\min }=m_{j}^{\min }+t-1$, and $m_{j}^{k}$ unchanged for $k \neq \max$, min. Otherwise repeat.

In the case where $P_{\text {octa }}$ is a triangle with each face containing exactly one vertex of $K$, the term of the disjunction (7.5) corresponding to the vertex of $K$ left outside the triangle plays no role in defining the cut, hence it can be dropped and the strengthening becomes simpler. This is even more true in the case of a cone, where only two terms of the disjunction are active. A particularly simple case is that of a "fixed shape" cone with apex at a vertex of $K$, and one face containing a side of $K$, the other face containing the diagonal of $K$. There are eight such cones, and every fractional $\left(f_{1}, f_{2}\right) \neq\left(\frac{1}{2}, \frac{1}{2}\right)$ (i.e. not lying on the diagonal of $K$ ) is strictly contained in four of them (see Fig. 6 in the next section).

We will illustrate the monoidal strengthening procedure on the conic cuts obtainable from these disjunctions. Here is a couple of them:

1. $\left(-x_{2} \geq 0\right) \vee\left(-x_{1}+x_{2} \geq 0\right)$

2. $\left(x_{2} \geq 1\right) \vee\left(-x_{1}-x_{2} \geq-1\right)$

or, after substituting $f_{i}+r^{i} s$ for $x_{i}, i=1,2$, 
1. $\left(-r^{2} s \geq f_{2}\right) \vee\left(\left(-r^{1}+r^{2}\right) s \geq f_{1}-f_{2}\right)$

2. $\left(r^{2} s \geq 1-f_{2}\right) \vee\left(\left(-r^{1}-r^{2}\right) s \geq f_{1}+f_{2}-1\right)$.

Each disjunction violated by the point $\left(f_{1}, f_{2}\right)$ has positive right-hand sides in each of its terms and gives rise to a valid cut $\alpha s \geq 1$, with coefficients $\alpha_{j}$ shown below, obtained by using multipliers normalized to yield a cut with a right-hand side of 1:

1. $\alpha_{j}=\max \left\{\frac{-r_{j}^{2}}{f_{2}}, \frac{-r_{j}^{1}+r_{j}^{2}}{f_{1}-f_{2}}\right\}$
2. $\alpha_{j}=\max \left\{\frac{r_{j}^{2}}{1-f_{2}}, \frac{-r_{j}^{1}-r_{j}^{2}}{f_{1}+f_{2}-1}\right\}$.

To apply the strengthening procedure, we note that for each term of the above disjunctions, the lower bound on the left-hand side of the inequality is just 1 unit less than the right-hand side, hence the difference between the latter and the former is exactly 1. Further, since each term has a single inequality, the vector $\theta^{k}$ of Theorem 7.1 becomes a scalar and can be set to 1 . The resulting strengthened coefficients for the above illustration are
1. $\hat{\alpha}_{j}=\min _{m_{j} \in M} \max \left\{\frac{-r_{j}^{2}+m_{j}^{1}}{f_{2}}, \frac{r_{j}^{1}-r_{j}^{2}+m_{j}^{2}}{f_{1}-f_{2}}\right\}$
2. $\hat{\alpha}_{j}=\min _{m_{j} \in M} \max \left\{\frac{r_{j}^{2}+m_{j}^{1}}{1-f_{2}}, \frac{-r_{j}^{1}-r_{j}^{2}+m_{j}^{2}}{f_{1}+f_{2}-1}\right\}$.

\section{Computational experiments}

Our initial computational experiments with cuts from two rows of the simplex tableau consisted in generating large numbers of cuts from all pairs of rows of the optimal simplex tableau, derived by solving the (CGLP) defined by (5.3), (5.4) for a multitude of objective functions, and examining the effect of adding those cuts to the optimal simplex tableau in addition to one round of GMI cuts. The results were utterly disappointing, in that the improvements obtained were insignificant. Our disappointment turned into surprise when we discovered that among a variety of cuts from quadrilaterals and triangles of various shapes, many of them facet-defining for conv $P_{D}$ and/or conv $P_{I}$, the best results were obtained with cuts from a specific fixed-shape family of triangles, which are almost never facet-defining. This family was that of triangles of type A, with three integer extreme points and one integer point in the interior of each face. So we focused our attention on this family. Further, since our main concern was with 0-1 programming, and conv $P_{D}^{=}$is a more constrained set than conv $P_{D}$, we designed a major computational experiment based on the use of two types of cuts for 0-1 programming, namely the above-described triangle cuts and a class of fixed-shape conical cuts.

We assess the strength of the cuts by analyzing the gap closed on instances from MIPLIB3_C_V2 (Margot 2010) when used in combination with standard Gomory cuts. MIPLIB3_C_V2 is a collection of 68 instances by Margot which are slight variations of the standard MIPLIB 3 (1996) and for which the validity of a candidate solution can be checked in finite precision arithmetic. We restricted the collection to a 

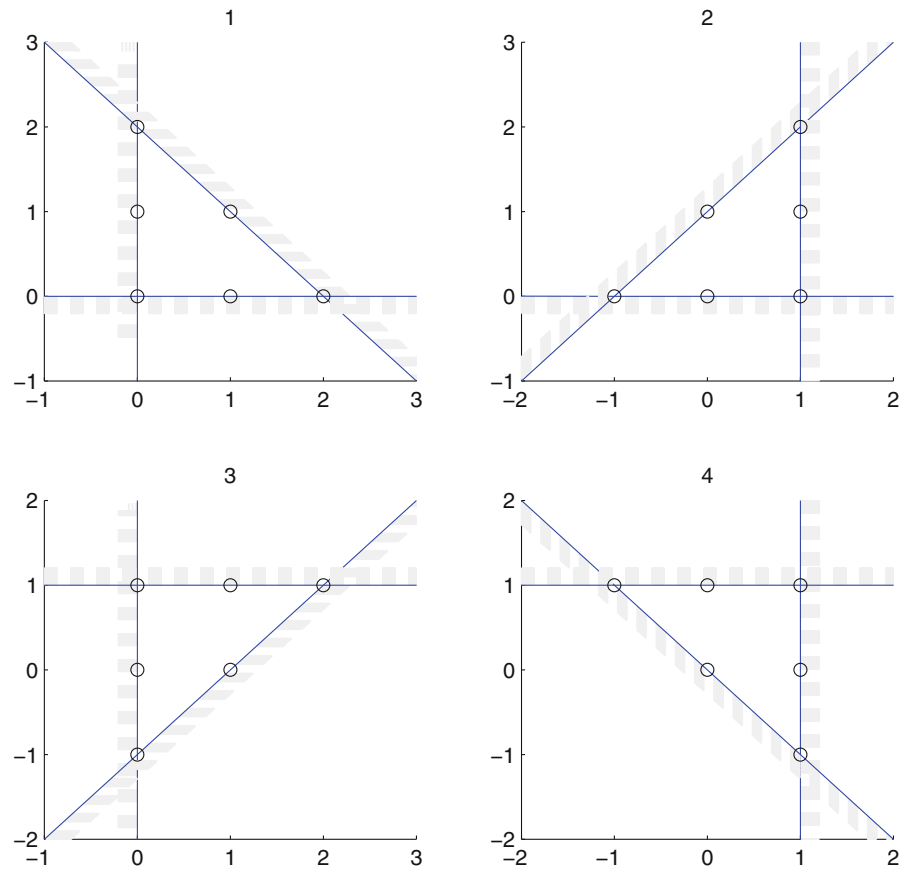

Fig. 5 Fixed shape Triangles $T_{A}$

subset of 41 instances. The considered instances are such that they contain at least two binary variables fractional in the optimal LP solution and the cut generation procedure on each round takes less than 3,600 s.

We generated the following two families of cuts

- Cuts from four triangles $T_{A}$ (shown in Fig. 5) whose vertices, expressed in terms of their $x_{1}, x_{2}$ coordinates, are:

- $(0,0) ;(2,0) ;(0,2)$

- $(-1,0) ;(1,0) ;(1,2)$

- $(0,-1) ;(2,1) ;(0,1)$

- $(1,-1) ;(1,1) ;(-1,1)$.

- Cuts from four of the eight cones of type $C_{A}$ (shown in Fig. 6):

- apex at $(0,0)$ and rays $(1,0),(1,1)$

- apex at $(0,0)$ and rays $(0,1),(1,1)$

- apex at $(0,1)$ and rays $(1,0),(1,-1)$

- apex at $(0,1)$ and rays $(0,-1),(1,-1)$

- apex at $(1,1)$ and rays $(-1,0),(-1,-1)$

- apex at $(1,1)$ and rays $(0,-1),(-1,-1)$

- apex at $(1,0)$ and rays $(-1,0),(-1,1)$

- apex at $(1,0)$ and rays $(0,1),(-1,1)$. 

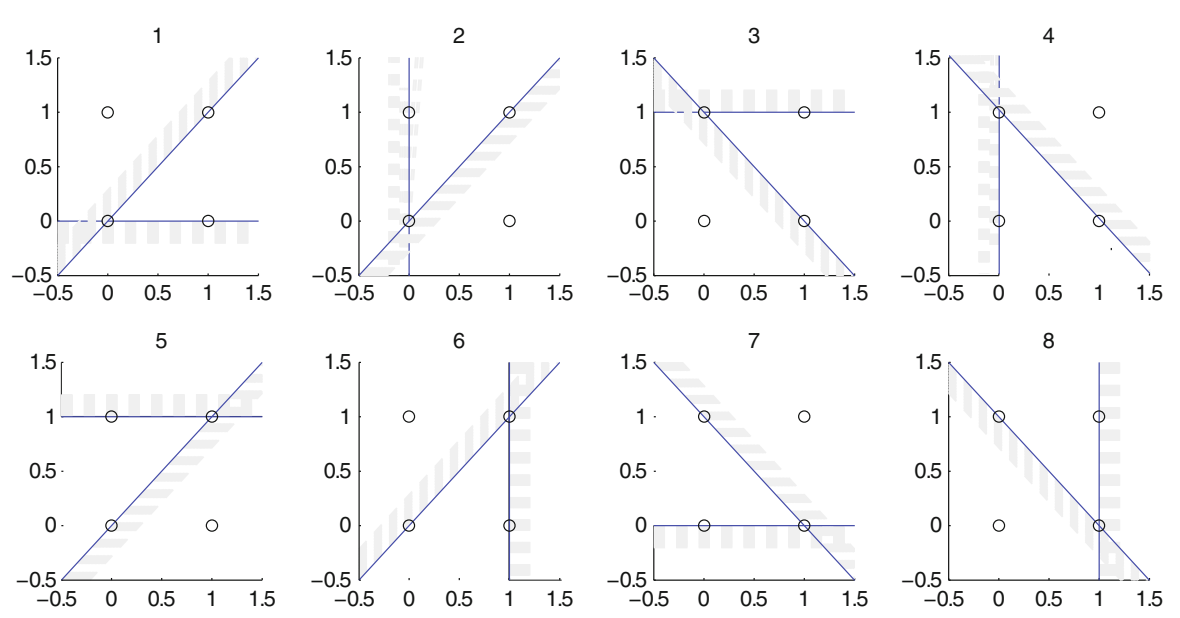

Fig. 6 Fixed shape cones $C_{A}$

The reason we only used four of these eight cones is that every $\left(f_{1}, f_{2}\right)$-pair is contained in four of these eight cones.

For each instance, we first solved the linear programming relaxation and generated a round of GMI cuts, a round being one cut from every row of the optimal simplex tableau associated with a binary basic variable with a fractional value. We then generated from each pair of rows with at least one fractional binary basic variable either (a) all cuts from the four triangles $T_{A}$, or (b) all cuts from four of the eight cones $C_{A}$ or both, and strengthened them via standard modularization as described in Sec. 6 (in case a) or monoidal strengthening as described in Sec. 7 (in case b).

We call this cut generating cycle a round. At the end of each round, we reoptimized the resulting linear program and removed all cuts that were not tight at the optimum. We generated up to five rounds of cuts for each instance.

The GMI cut generator we used is the Gomory Two, developed by Margot (2009) and available at http://wpweb2.tepper.cmu.edu/fmargot/CODE/cutgentest.tar.gz. In generating our triangle and conic cuts we used the same tolerances and parameter values as for Gomory Two, namely those of "Margot's variant G2P1" [see page 15 of Margot (2009)]. Tables 1, 2, 3, 4 summarize the results of our experiments with these cuts. Table 1 shows the outcome of applying all three types of cuts in the above-described manner, with strengthening, for one round. Column 1 lists the 41 test instances mentioned above. Column 2 shows the percentage of the integrality gap closed by one round of GMI cuts, while the next two columns show the number of cuts generated and added to the LP relaxation, along with the number of cuts deleted after reoptimization as nonbinding. The next three columns show the same data (i.e. percentage of gap closed and number of cuts added, respectively deleted) after generating a cut from each of the four triangles $T_{A}$ associated with every pair of basic $0-1$ variables with at least one fractional member, and a cut from each of the eight cones associated with every such pair, provided the cone contains such a pair in its interior. The column "Improvement in gap closed" shows the percentage improvement in the integrality 


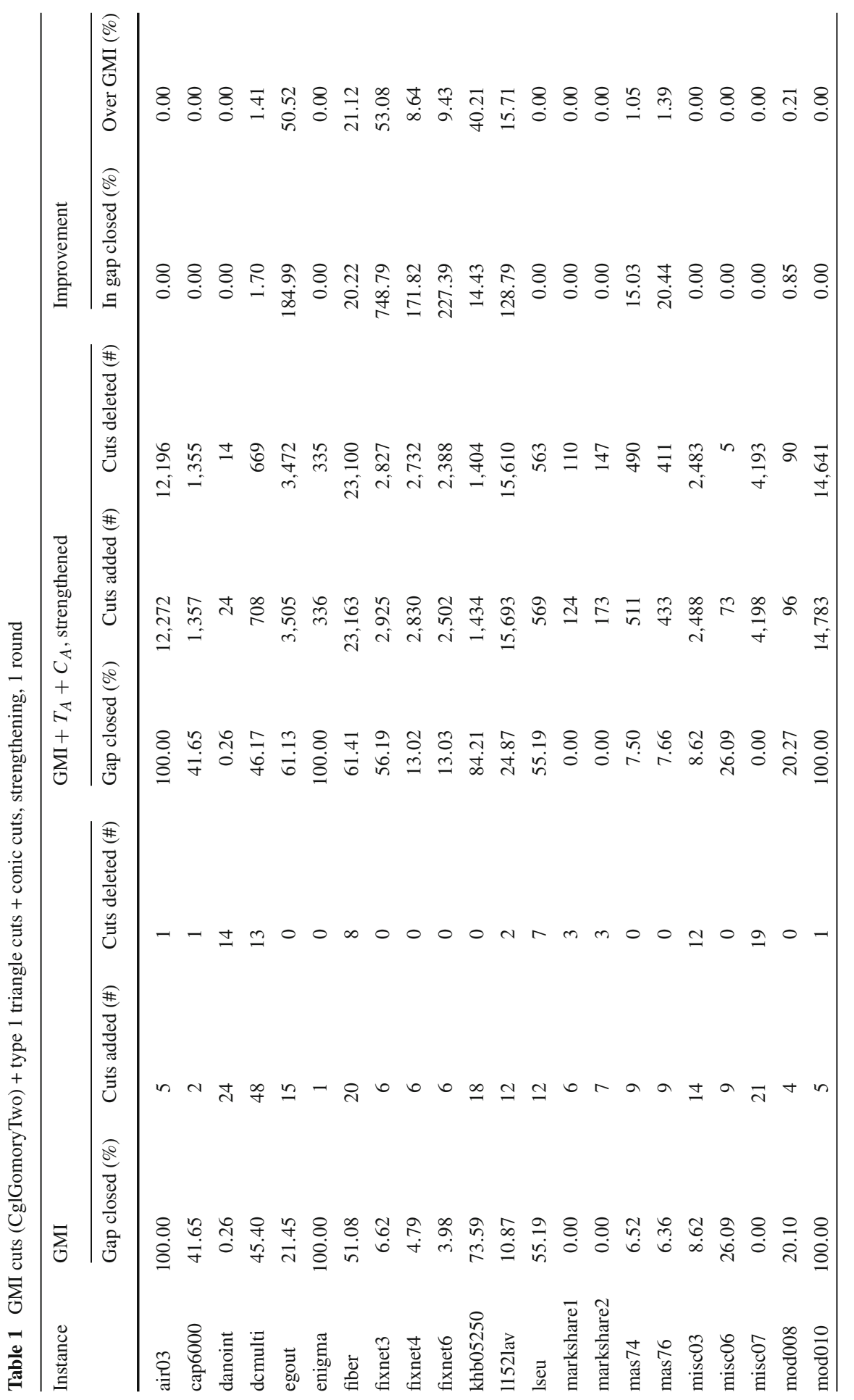




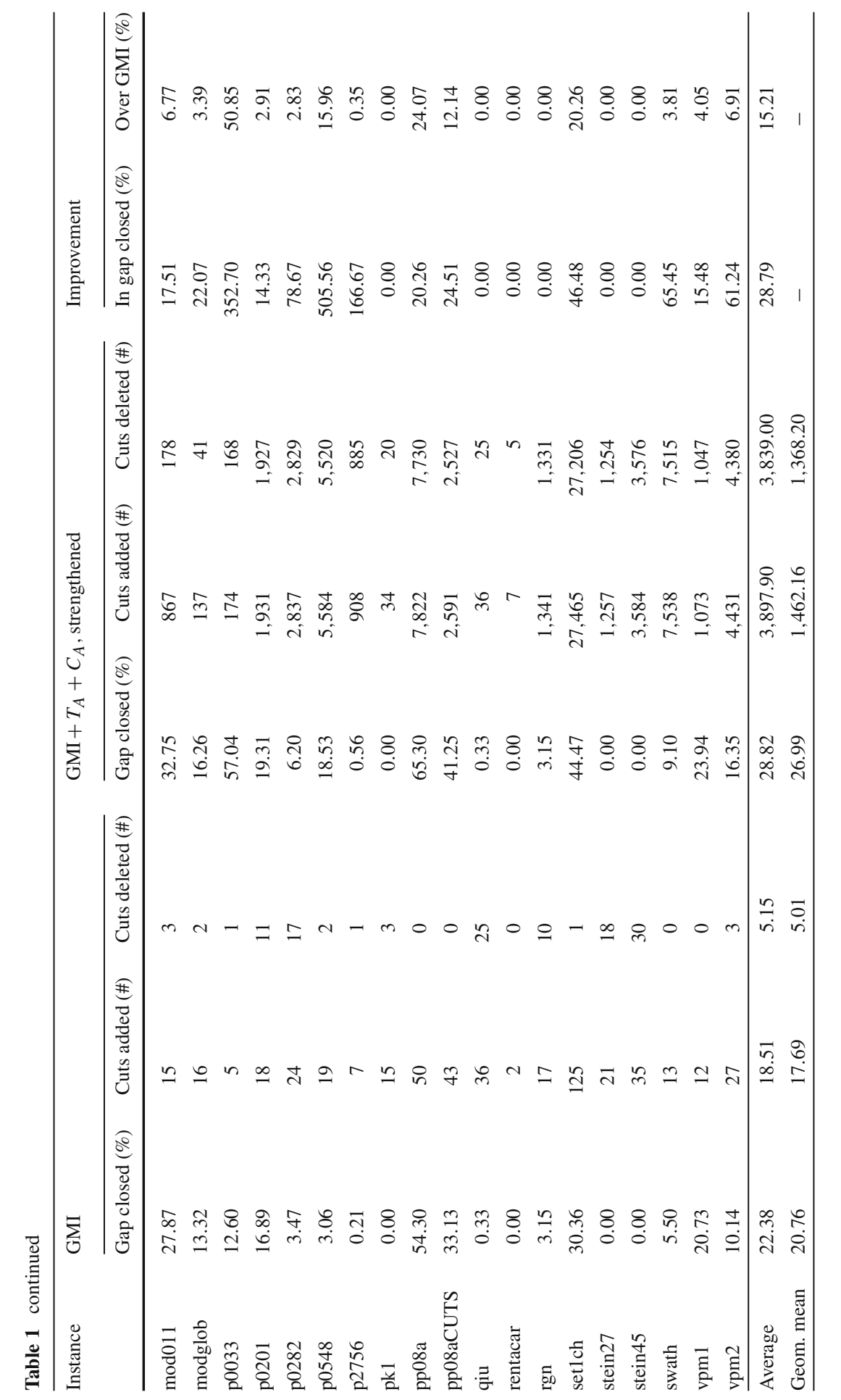




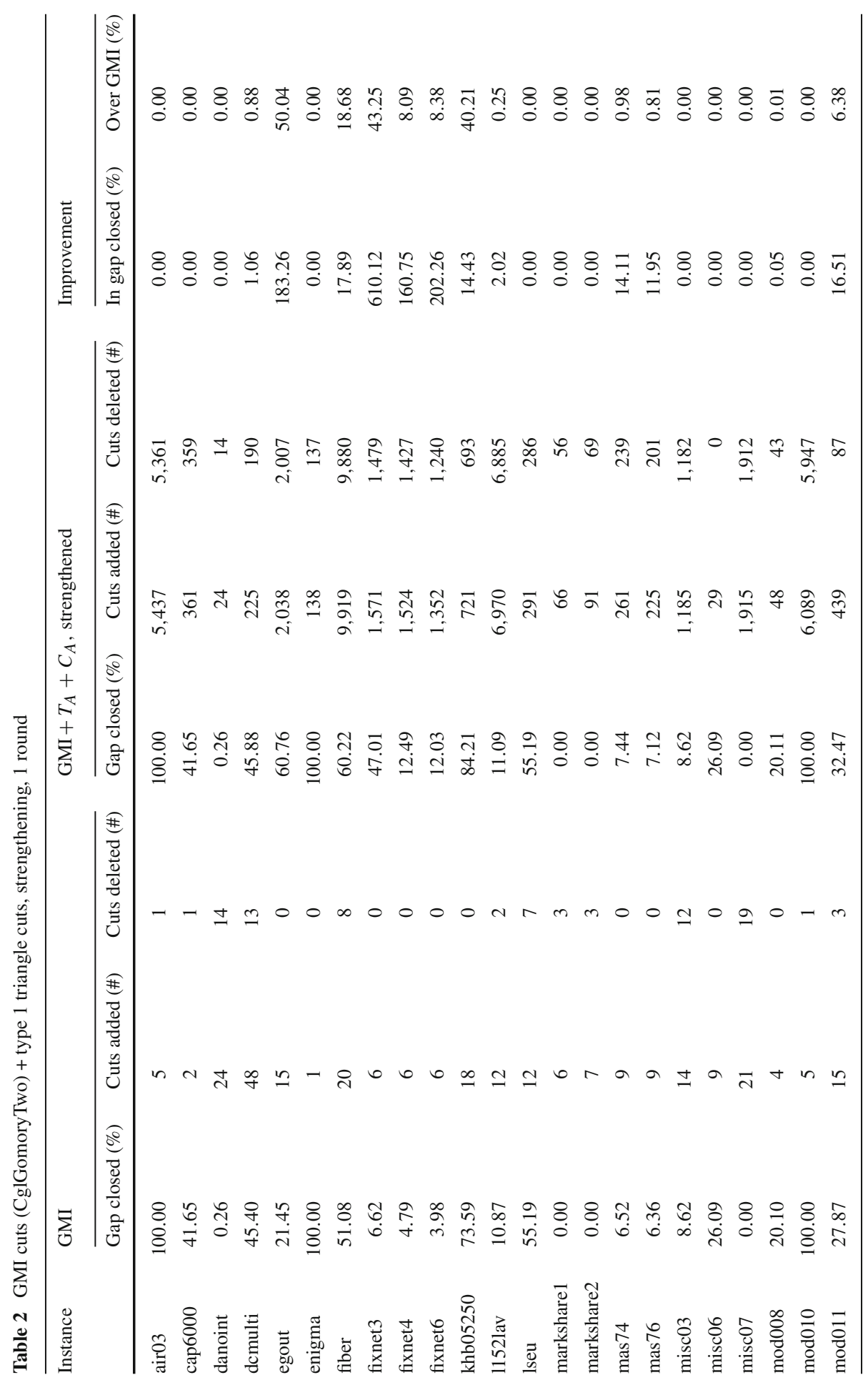




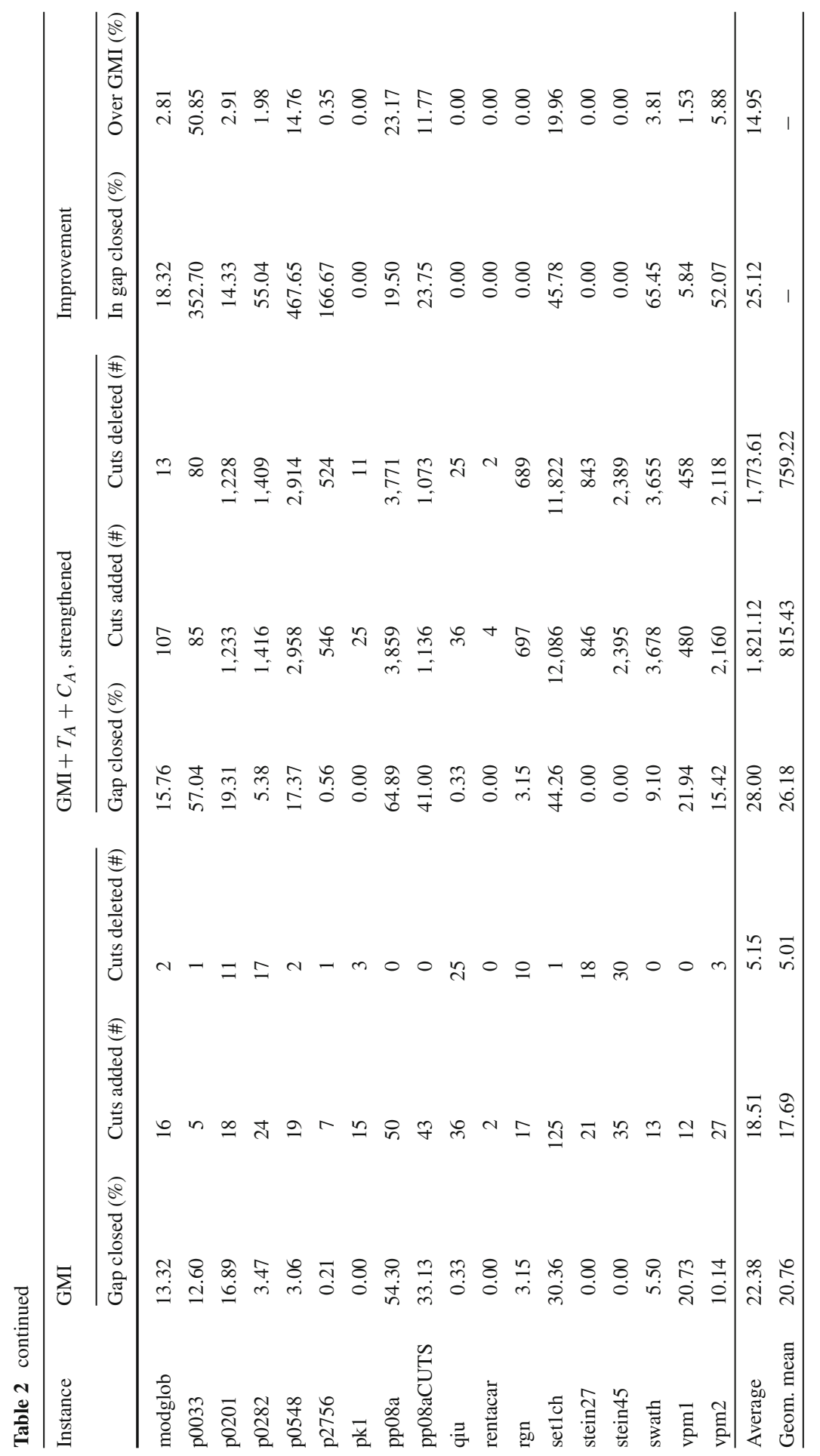




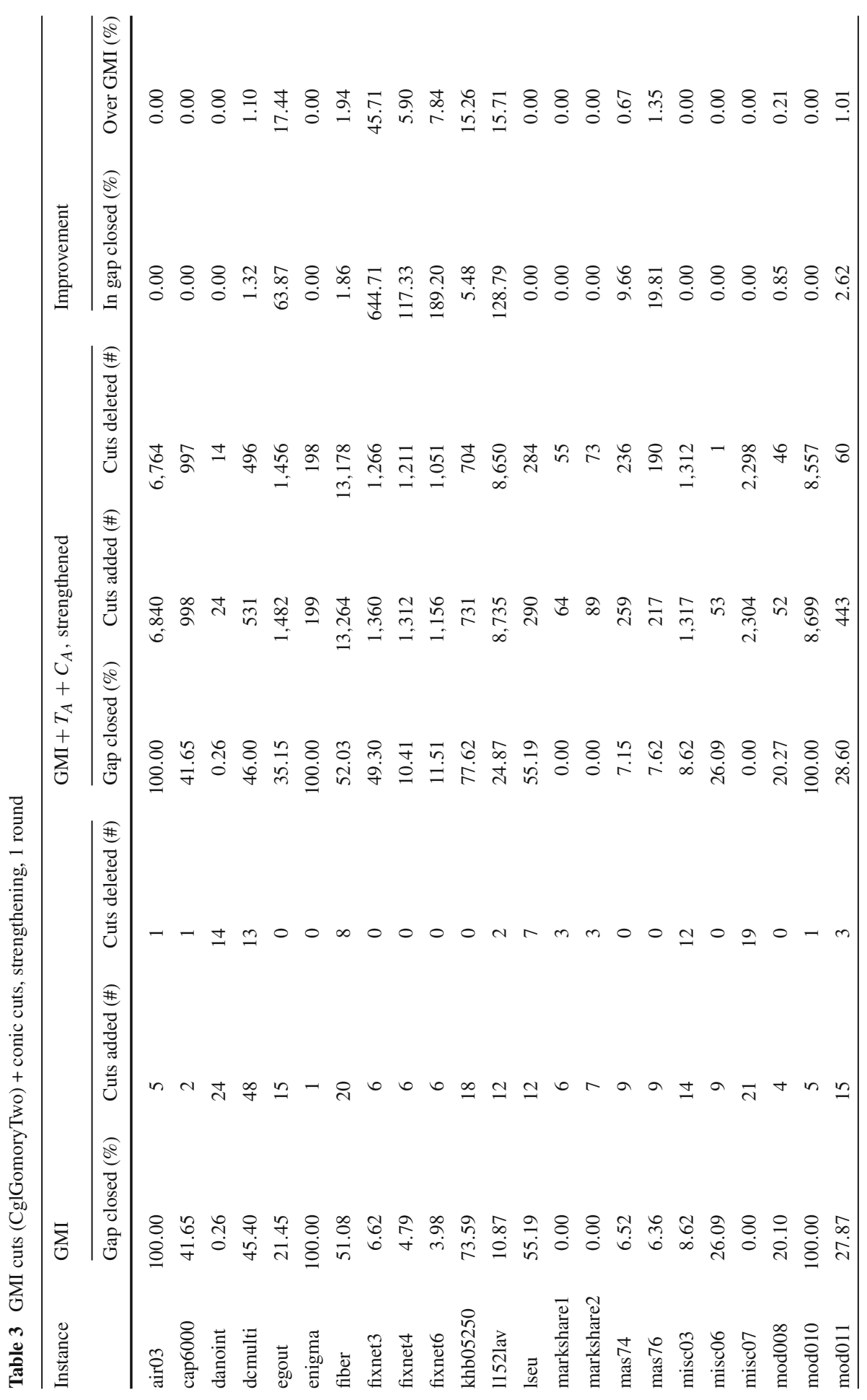




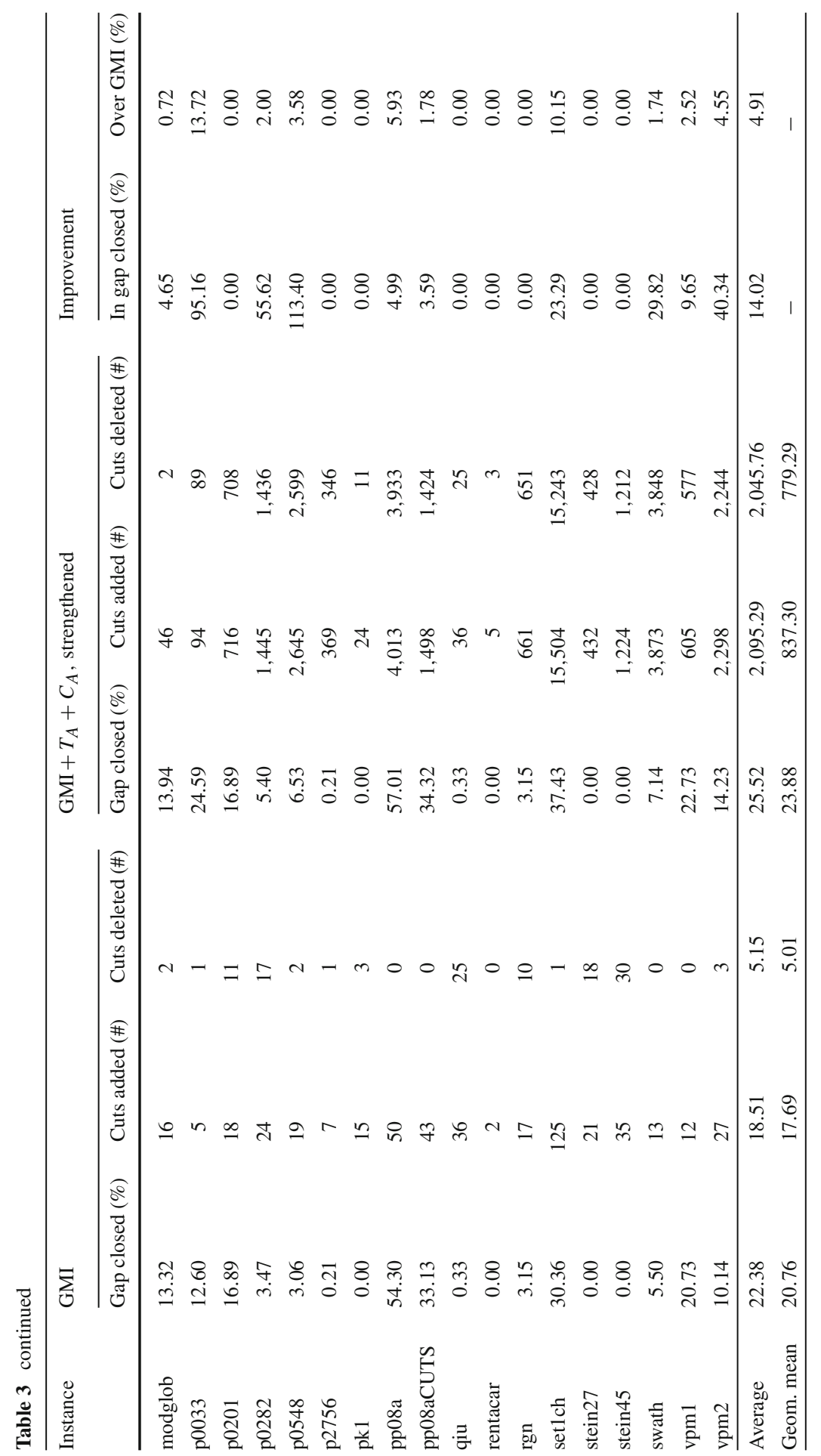


Table 4 CglGomoryTwo + type 1 triangles + conic cuts, strengthening, 5 rounds

\begin{tabular}{|c|c|c|c|c|c|c|}
\hline \multirow[t]{2}{*}{ Round } & \multicolumn{2}{|c|}{ GMI gap \% } & \multicolumn{2}{|c|}{$\mathrm{GMI}+T_{A}+C_{A}$, str. gap $\%$} & \multicolumn{2}{|c|}{ Improvement $\%$} \\
\hline & Average & Geom. mean & Average & Geom.mean & In gap closed & Over GMI \\
\hline 1 & 22.38 & 20.76 & 28.82 & 26.99 & 28.79 & 15.21 \\
\hline 2 & 28.22 & 26.33 & 33.98 & 32.02 & 20.41 & 9.37 \\
\hline 3 & 31.07 & 29.01 & 36.68 & 34.62 & 18.05 & 6.85 \\
\hline 4 & 32.83 & 30.71 & 37.78 & 35.68 & 15.07 & 6.40 \\
\hline 5 & 34.61 & 32.37 & 38.86 & 36.70 & 12.29 & 7.79 \\
\hline
\end{tabular}

gap closed by all three types of cuts versus the GMI cuts alone computed as

$$
100 \frac{\operatorname{gap}_{\mathrm{GMI}+T_{A}+C_{A}}-\text { gap }_{\mathrm{GMI}}}{\operatorname{gap}_{\mathrm{GMI}}} .
$$

The column "Improvement over GMI" gives the percentage of gap left by GMI cuts closed by adding the other two types of cuts, computed as

$$
100 \frac{\mathrm{LP}_{\mathrm{GMI}+T_{A}+C_{A}}-\mathrm{LP}_{\mathrm{GMI}}}{\mathrm{IP}_{\mathrm{OPT}}-\mathrm{LP}_{\mathrm{GMI}}}
$$

where $\mathrm{LP}_{\mathrm{GMI}}$ is the LP bound obtained by adding GMI cuts only, $\mathrm{LP} \mathrm{GMI}_{\mathrm{G}}+\mathrm{T}_{\mathrm{A}}+\mathrm{C}_{\mathrm{A}}$ is the LP bound obtained by adding all cuts, $\mathrm{IP}_{\mathrm{OPT}}$ is the value of the optimal integer solution. The last two rows of the table report the arithmetic average and the shifted geometric mean ( shift parameter $=200)$.

As the table shows, the integrality gap closed on average, which is $22.38 \%$ in the case of the GMI cuts, reaches $28.82 \%$ when the two remaining types of cuts are added, an increase of $28.79 \%$. The number of triangle cuts and conical cuts generated is of course much larger than that of GMI cuts. While the latter is bounded by the number of basic 0-1 variables fractional at the optimum, in case of the other two types of cuts this number is bounded by 8 times the number of pairs of basic $0-1$ variables with at least one fractional member. From the table it is clear that after reoptimization few of the added cuts remain active (about $2 \%$ ), while the rest is removed. The table also reveals marked differences in the impact of the two-row cuts on different instances, from 0 impact in about $40 \%$ of the instances, to a more than sevenfold increase of the gap closed in the highest-impact case.

Tables 2, 3 show the effect of using only triangle cuts or only conic cuts on top of the GMI cuts. On average, using only triangle cuts has a bigger effect than using only conic cuts (on top of the GMI cuts). Clearly, the joint effect of using both types of cuts is substantially stronger than is the case with a single type.

Finally, Table 4 shows the effect of generating both types of two-row cuts on top of GMI cuts, as in Table 1, but this time for 5 rounds instead of just 1 .

We also ran experiments with a different GMI cut generator, namely the CoinCglGomory routine of the $\mathrm{Cgl}$ package of COIN-OR (COIN-OR 2000). The outcome of these runs is given in Tables 5, 6, which show a bigger advantage for adding the two-row 


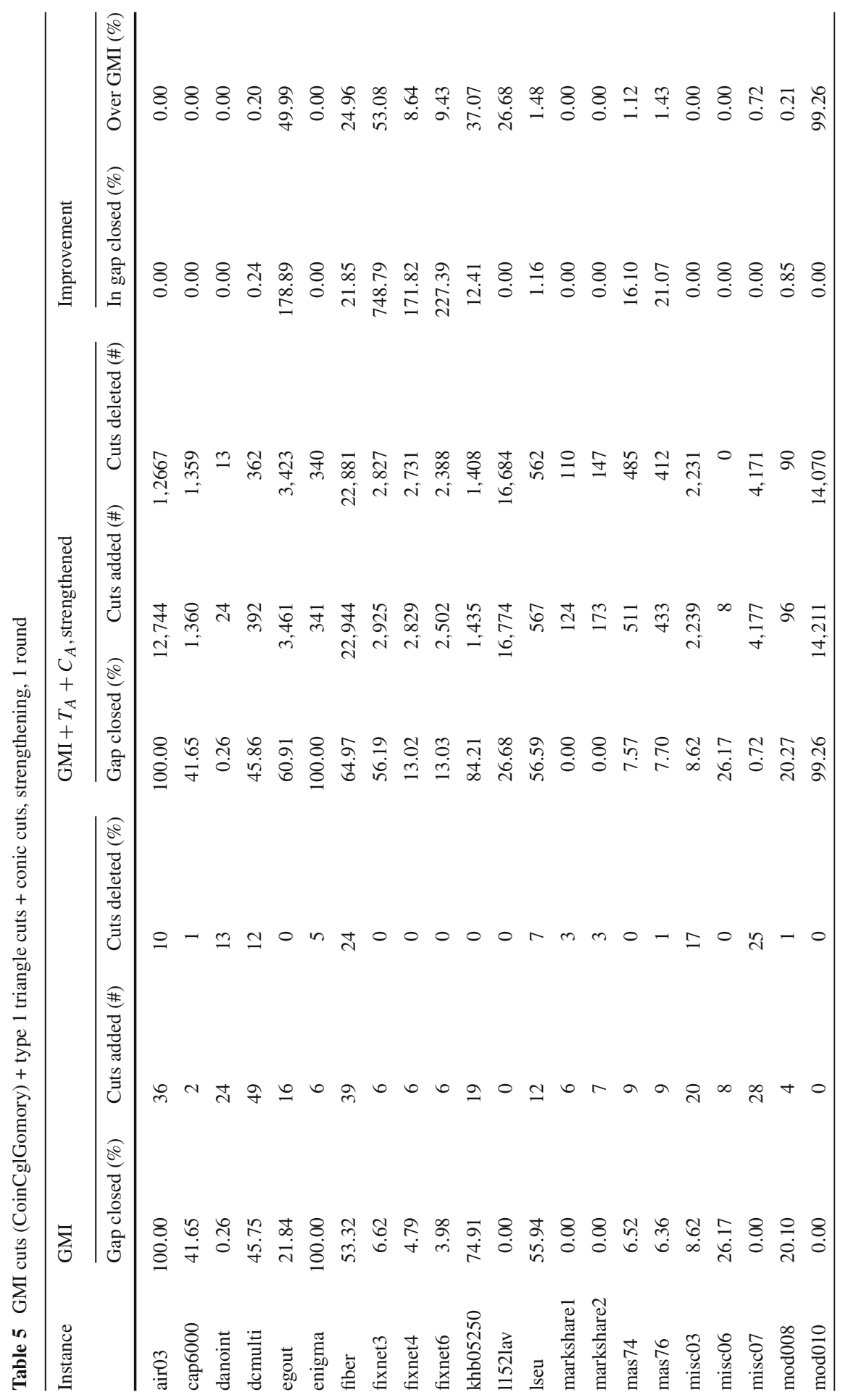




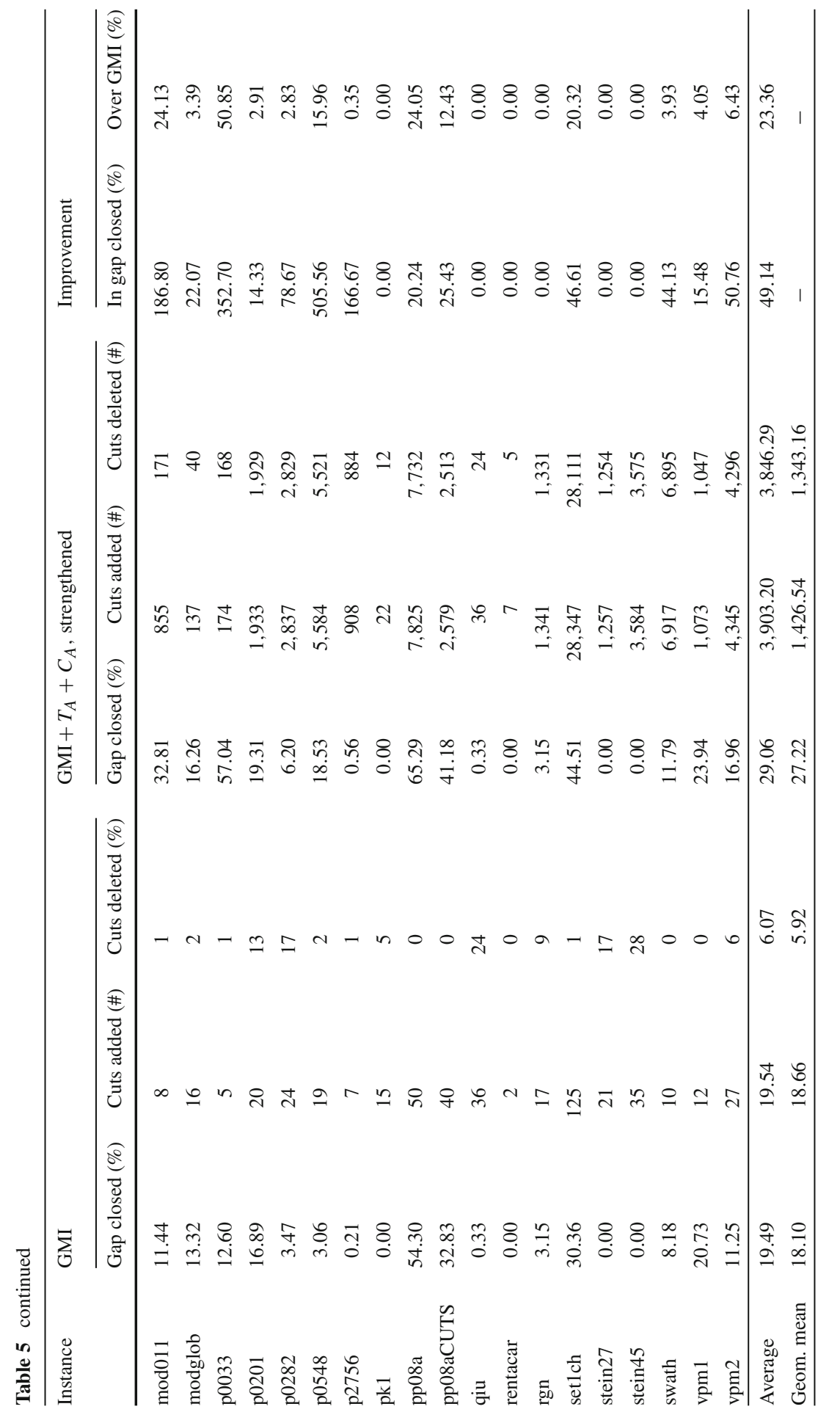


Table 6 CoinCglGomory + type 1 triangles + conic cuts, strengthening, 5 rounds

\begin{tabular}{|c|c|c|c|c|c|c|}
\hline \multirow[t]{2}{*}{ Round } & \multicolumn{2}{|c|}{ GMI gap (\%) } & \multicolumn{2}{|c|}{$\mathrm{GMI}+T_{A}+C_{A}$, str. gap (\%) } & \multicolumn{2}{|c|}{ Improvement (\%) } \\
\hline & Average & Geom.mean & Average & Geom.mean & In gap closed & over GMl \\
\hline 1 & 19.49 & 18.10 & 29.06 & 27.22 & 49.14 & 23.36 \\
\hline 2 & 24.94 & 23.18 & 34.01 & 32.08 & 36.38 & 18.56 \\
\hline 3 & 27.87 & 25.86 & 36.70 & 34.65 & 31.67 & 19.09 \\
\hline 4 & 29.57 & 27.41 & 37.95 & 35.84 & 28.31 & 21.67 \\
\hline 5 & 30.48 & 28.24 & 38.78 & 36.67 & 27.24 & 21.09 \\
\hline
\end{tabular}

cuts than the previous tables. The CoinCglGomory cut generator is less aggressive than Gomory Two and produces slightly weaker bounds which explains the difference in the results.

Recently, due to the work of Margot (2009), it has become possible to submit MIP cut generators to rigorous reliability tests. The codes used in our experiments were submitted to these tests. The improvement given by the cuts after one round were found to be statistically significant with a 99\% confidence level. See Margot (2009) for details.

On the whole, these experiments with triangle and conical cuts for mixed 0-1 programs are somewhat encouraging, in that they show a potential for significant improvements over GMI cuts from the use of cuts from multiple rows. Our experience with cone cuts is the first of its kind, so we cannot compare it to others. On the other hand, triangle cuts have been tested also by other researchers; in particular the paper by Basu et al. (2010) describes a large, carefully planned and executed experiment which reports little improvement from adding triangle cuts of various types on top of GMI cuts. We have at least a partial explanation for this seeming discrepancy. Namely, the experiments in Basu et al. (2010) are for the special case of cuts from two rows of the simplex tableau, only one of which has a fractional (integer-constrained) basic variable, the other one having an integer-valued $f_{i}$. Furthermore, among all such pairs, the authors select a small subset based on four different criteria, aiming at judiciously choosing a number of triangle cuts roughly equal to the number of GMI cuts generated from the same tableau. Our procedure, by contrast, generates a large number of triangle cuts, of the order of the square of the number of GMI cuts from the same tableau. Apparently using such a large number of cuts pays off in terms of the integrality gap closed, although most of the cuts used end up being slack at the optimum.

\section{References}

Andersen K, Louveaux Q, Weismantel R, Wolsey LA (2007) Cutting planes from two rows of a simplex tableau. In: IPCO 12, Lecture notes in computer science, vol 4513. Springer, Berlin, pp 1-15

Balas E (1971) Intersection cuts - a new type of cutting planes for integer programming. Oper Res 19:19-39

Balas E (1998) Disjunctive programming: properties of the convex hull of feasible points. Discret Appl Math 89:3-44

Balas E (1979) Disjunctive programming. Ann Discret Math 5:3-51 
Balas E (2009a) Intersection cuts from maximal lattice-free convex sets and lift-and-project cuts from multiple-term disjunctions. American Mathematical Society Western Section Spring Meeting, San Francisco, April, pp 25-27

Balas E (2009b) Multiple-term disjunctive cuts and intersection cuts from multiple rows of the simplex tableau. In: 20th international symposium on mathematical programming, Chicago, August 23-28, 2009

Balas E, Ceria S, Cornuéjols G (1993) A lift-and-project cutting plane algorithm for mixed 0-1 programs. Math Program 58:295-324

Balas E, Ceria S, Cornuéjols G (1996) Mixed 0-1 programming by lift-and-project in a branch-and-cut framework. Manag Sci 42:1229-1246

Balas E, Jeroslow R (1980) Strengthening cuts for mixed integer programs. Eur J Oper Res 4:224-234

Balas E, Perregaard M (2003) A precise correspondence between lift-and-project cuts, simple disjunctive cuts, and mixed integer Gomory cuts for 0-1 programming. Math Program 94:221-245

Basu A, Bonami P, Cornuéjols G, Margot F (2009) On the relative strength of split triangle and quadrilateral cuts. Math Program 126:1220-1229

Basu A, Bonami P, Cornuéjols G, Margot F (2010) Experiments with Two-Row Cuts from Degenerate Tableaux. INFORMS J Comput 23(4): 578-590

Borozan V, Cornuéjols G (2009) Minimal valid inequalities for integer constraints. Math Oper Res 34:538-546

COmputational INfrastructure for Operations Research (COIN-OR) (2000) http://www.coin-or.org

Conforti M, Cornuéjols G, Zambelli G (2011) Corner polyhedron and intersection cuts. Surv Oper Res Manag Sci 16:105-120

Cook W, Kannan R, Schrijver A (1990) Chvatal closures for mixed integer programming problems. Math Program 47:155-174

Cornuéjols G, Margot F (2009) On the facets of mixed integer programs with two integer variables and two constraints. Mathe Program A 120:429-456

Dash S, Dey S, Gunluk O (2010) Two dimensional lattice-free cuts and asymmetric disjunctions for mixedinteger polyhedra. Math Program A. doi:10.1007/s/0/07-011-0455-1

Dey S, Wolsey LA (2010) Two row mixed-integer cuts via lifting. Math Program B 124:143-174

Dey S, Lodi A, Tramontani A, Wolsey L (2010) Experiments with two-row tableau cuts. In: IPCO 14, Lecture notes in computer science, vol 6080. Springer, 2010, pp 424-437. doi:10.1007/978-3-642-13036-6

Gomory RE (1960) An algorithm for the mixed integer problem. RM-2597. The Rand Corporation

Gomory RE (1969) Some polyhedra related to combinatorial problems. J Linear Algebra Appl 2:451-458

Johnson EL (1974) On the group problem for mixed integer programming. Math Program Study 3:137-179

Margot F (2010) MIPLIB3-C-V2.http://wpweb2.tepper.cmu.edu/fmargot/MPS/miplib3-c-v2.tar.gz

Margot F (2009) Testing cut generators for Mixed-Integer Linear Programming. Math Program Comput 1:69-95

MIPLIB 3 (1996) Available at http://www.caam.rice.edu/ bixby/miplib/miplib3.html

Qualizza A (2011) Cutting planes for mixed integer programming. Dissertation, Tepper School of Business, Carnegie Mellon University 